\title{
SIMBOLO, MIRACOLO, TENTAZIONE. IL CIBO NELLA LETTERATURA AGIOGRAFICA MEDIEVALE PRIMA E DOPO IL SECOLO XII
}

\author{
GIOVANNI PAOLO MAGGIONI (*)
}

SUNTO. - La religione cristiana è caratterizzata da un rapporto ambivalente con il cibo. Da una parte, la liturgia è imperniata sul pane e il vino, corpo e sangue del Cristo; dall'altra, il corpo, che si è costretti a nutrire e a curare, è stato spesso visto come un intollerabile fardello sulla via della salvezza. Pur in questa ambivalenza, nella letteratura agiografica del primo Medioevo sembra essere predominante una concezione negativa del cibo, il tramite di ogni vizio, a cui l'uomo non può rinunciare, mentre dal XII secolo si afferma una nuova concezione di corpo e, conseguentemente, una diversa considerazione del cibo e dell'alimentazione. Vengono proposti qui alcuni esempi riguardanti gli alimenti primari, tra cui il pane, il vino e il latte, che illustrano questa evoluzione.

$$
* * *
$$

ABSTRACT. - The Christian religion is characterized by an ambivalent relationship with food. On the one hand, the liturgy is centered on the bread and wine, body and blood of Christ; on the other, the body, that you are forced to feed, has often been seen as an intolerable burden on the path of salvation. Despite this ambivalence, in the hagiographic literature of the early Middle Ages seems to be predominantly a negative conception of food, the trámite of every vice, to which man can not give up, while the twelfth century dominates a new idea of the body and, consequently, a different consideration of food and nutrition. Some hagiographic exempla regarding the primary foods, including bread, wine and milk, illustrate this evolution.

Occuparsi di santi medievali vuol dire occuparsi di figure agiografiche che costellano un periodo di tempo più che millenario, in cui si è

(*) Università degli Studi del Molise, Italia. E-mail: giovanni.maggioni@unimol.it 
passati da leggende esemplari imperniate semplicemente attorno a un' $i$ mitatio Christi basata sul martirio a una figura come quella di Francesco d'Assisi, estremamente complessa e per certi versi rivoluzionaria. Per l'appunto la figura agiografica di Francesco, il cui corpo vivente è stato santificato in una speciale imitazione di Cristo segnata dalle stimmate, è il coronamento di una nuova concezione di corpo - e quindi di piacere, di peccato, nel nostro caso la gola - affermatasi a partire dal XII secolo ed evidente anche nei testi agiografici.

Il mutare dei modelli agiografici corre parallelo ai cambiamenti che hanno attraversato la società e la cultura nel millennio medievale. Le diverse componenti che hanno parte nella composizione del racconto agiografico presuppongono infatti una interazione reciproca con la cultura in cui le legendae sanctorum vengono composte: all'esistenza (non sempre reale) del santo e della sua vicenda terrena fa seguito lo sviluppo di un culto religioso e infine la composizione di un racconto agiografico che propone quella vicenda terrena (o la crea) rielaborandola in un'essenzialità esemplare, che soggiace ai modelli culturali imperanti al tempo. C'è dunque un tempo (e un luogo) in cui il testo agiografico viene scritto, ma ci sono altri tempi e altri luoghi in cui il testo agiografico viene fruito, ovvero letto, spiegato, predicato, in diversi momenti interpretativi e con differenti fruizioni. C'è la lettura nel silenzio del refettorio, sottoposta al silenzio dei monaci, e c'è l'interpretazione data al testo agiografico proveniente da un pulpito per bocca di un predicatore che utilizza una figura agiografica, magari risalente a un millennio prima, per trasmettere efficacemente contenuti etici e dottrinali per lui attuali. Ci sono poi santi ammirabili e ci sono santi imitabili.

In questa sede vorrei dar conto di questa evoluzione e del cambiamento dei modelli agiografici attraverso il raffronto di alcuni temi della letteratura agiografica riguardanti appunto il cibo, il piacere, il peccato di gola, dai primi secoli medievali fino al XIII -XIV secolo ${ }^{1}$.

1 Queste pagine sono pesantemente debitrici degli studi di Carla Casagrande e Silvana Vecchio, soprattutto Casagrande-Vecchio 2000, 124-148 e Vecchio 2009, 27 39. Fondamentale è stata inoltre la lettura di Montanari 2015 e, per la documentazione riguardante Francesco d'Assisi, Casio-Messa 2015. 


\section{Il CIBO ELEMENTO FONDANTE DELLA RITUALITÀ CRISTIANA, MA ANCHE OCCASIONE DI PECCATO}

Qui delectationes corporales desiderant et animas suas fame mori permittunt, similes sunt cuidam homini qui, dum a facie unicornis ne ab eo deuoraretur uelocius fugeret, in quodam baratrum magnum cecidit. Dum autem caderet, manibus arbustulam quandam apprehendit et in base quadam lubrica et instabili pedes fixit. Respiciens uero uidit duos mures, unum album et alium nigrum, incessanter radicem arbustule quam apprehenderat corrodentes et iam prope erat ut ipsam absciderent. In fundo autem baratri uidit draconem terribilem spirantem ignem et aperto ore ipsum deuorare cupientem, super basem uero, ubi pedes tenebat, uidit quatuor aspidum capita inde prodeuntia. Eleuans autem oculos uidit exiguum mellis de ramis illius arbustule oblitusque periculi in quo undique positus erat, se ipsum dulcedini illius modici mellis totum dedit. ${ }^{2}$

Si è riproposto qui un brano tratto dalla storia testuale della Vita di Barlaam e Josaphat, un testo agiografico che interpreta a suo modo la leggenda di Buddha. Il testo greco, probabilmente del X secolo, ma attribuito a Giovanni Damasceno, ebbe due traduzioni in ambito latino e si diffuse in Europa occidentale soprattutto a partire dal XII secolo ${ }^{3}$. Si tratta in realtà di un testo dalla lunga e complicata tradizione ${ }^{4}$ : in Italia la leggenda è conosciuta soprattutto nella traduzione del 1979 di una raccolta inglese del 1939 di racconti zen giapponesi di varia origine 5 , ma risale al

2 Vita sanctorum Barlaam eremitae et Josaphat Indiae regis, XII, PL 73, col. 493, citata qui nella rielaborazione fattane da Iacopo da Varazze nella Legenda aurea, CLXXVI 96-112: «Un tale fuggendo a gran velocità di fronte a un unicorno, per non esserne divorato, finì in un burrone profondo. E mentre precipitava si aggrappò con le mani a un alberello appoggiando i piedi su una sporgenza scivolosa e instabile. Sporgendo il collo vide due topi, uno bianco e uno nero, che rodevano senza sosta la radice dell'arbusto al quale si era aggrappato, ed erano ormai sul punto di reciderla. In fondo al precipizio vide un drago terribile che sputava fuoco e che aspettava di divorarlo standosene con la bocca aperta, mentre sulla sporgenza su cui teneva i piedi vide spuntare le teste di quattro vipere. Alzando gli occhi vide un po' di miele sui rami dell'alberello e dimenticandosi dei pericoli dai quali era circondato da ogni lato, si abbandonò con tutto se stesso alla dolcezza di quel po' di miele» (Stella 2007, 1396-1397).

3 Sulla tradizione latina della leggenda, cfr. Chiesa 1983, 521-44 e Dapelo 2001, 179-220.

4 Per un quadro d'insieme si veda l'introduzione di Ronchey 2012, vii-cvii.

5 Senzaki-Reps 1973. 
Mahabharata e ha un fortleben anche in Tolstoi ${ }^{6}$, passando per la rappresentazione dell'albero della vita nel battistero di Parma [cfr. Fig. 1].

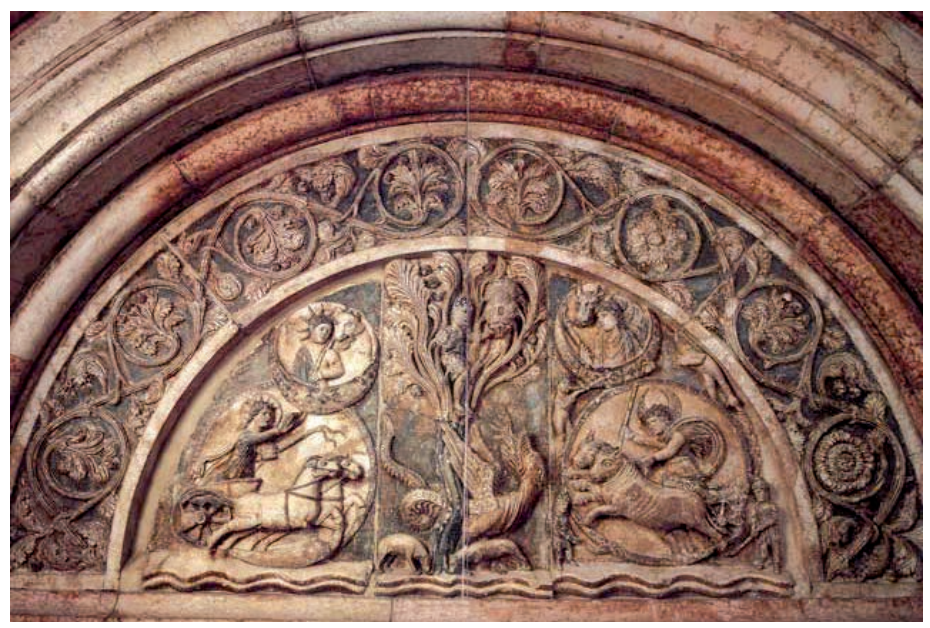

Fig. 1

La spiegazione dell'aneddoto proposta dai predicatori medievali sembra inequivocabile, poiché assaporare la dolcezza del miele ha un valore negativo e significa essere traviati e distolti dalla via della salvezza. Così ad esempio Iacopo da Voragine nella sua Legenda aurea:

Vnicornis autem mortis tenet figuram que hominem semper persequitur et apprehendere cupit, baratrum uero mundus est omnibus malis plenus. Arbustula uniuscuiusque uita est que per horas diei et noctis quasi per murem album et nigrum incessanter consumitur et incisioni appropinquat. Basis quatuor aspidum corpus ex quatuor elementis compositum, quibus inordinatis corporis compago dissoluitur. Draco terribilis os inferni cunctos deuorare cupiens, dulcedo ramusculi delectatio fallax mundi, per quam homo seducitur ut periculum suum minime intueatur. ${ }^{7}$

6 Tolstoj 2010, cap. 4.

7 Jacobus de Voragine, Legenda aurea, CLXXVI 119-122: «L'unicorno rappresenta la morte, che insegue sempre l'uomo e cerca di prenderlo; il burrone è invece il mondo, colmo di ogni male. L'arbusto è la vita di ognuno di noi, che viene incessantemente consumata dalle ore del giorno e della notte come da un topo bianco e da uno 
Nonostante le apparenze, durante il millennio medievale il rifiuto del cibo e del piacere che ne può derivare non è stato però uniforme e costante nei testi agiografici. I cambiamenti delle condizioni religiose, socio-economiche, politiche, climatiche hanno indubbiamente influito sulla rappresentazione dei modelli di santità. Vi è stata dunque un'evoluzione culturale che ha visto cambiare le concezioni di corpo e, conseguentemente, la considerazione del cibo, visibile anche nella letteratura agiografica dove dopo il XII secolo è evidente un rapporto diverso verso il cibo e l'alimentazione da parte dei protagonisti delle legendae sanctorum.

Certo i modelli agiografici dei primi secoli, che erano soprattutto martiri o eremiti, non hanno favorito alcuna indulgenza verso il cibo e verso quel vizio, la gola, considerata come la porta d'entrata di ogni altra nefandezza, sull'esempio di sant'Antonio eremita il quale

Mangiava una volta al giorno, dopo il tramonto del sole, talora ogni due giorni, e talora ogni quattro giorni. Il suo cibo era di pane e sale, e beveva solo acqua. È superfluo parlare del vino e delle carni, poiché nemmeno altri inferiori a lui in virtù mangiavano queste cose. ${ }^{8}$

La leggenda di sant'Antonio e quelle dei Padri del deserto diffusesi con le Vitae Patrum raccontavano infatti di personaggi andati oltre i limiti e le necessità naturali della loro condizione di uomini fino a porsi come intermediari tra l'umano e il divino, fino a rinunciare alla vita: un contesto che sembra presupporre un'idea completamente negativa del cibo e un conseguente rifiuto che ha ispirato alcune esperienze del monachesimo orientale. Inoltre l'astinenza dal cibo era vista come una necessaria penitenza, in quanto implicava una mortificazione del

nero, e si avvicina al punto in cui sarà recisa. La sporgenza con le quattro vipere rappresenta il corpo, composto dei quattro elementi: nel loro disordine la compattezza del corpo si dissolve. Il drago terribile è la bocca dell'inferno che ha brama di divorare tutti; la dolcezza del ramoscello l'ingannevole piacere del mondo, dal quale l'uomo si fa sedurre senza badare più al suo pericolo» (Stella 2007, 1396-1399).

8 Athanasius, Vita Antonii: «Manducabat enim semel in die, post occasum solis, aliquando post biduum, et aliquoties post quadriduum gustabat. Erat enim illi esca panis et sal, et potus sola aqua. De vino enim et de carnibus superfluum est dicere, quando nec apud alios qui inferiores illo erant in virtute aliquid tale inveniebatur» (Citati-Lilla 1974, 22-23). 
corpo materiale che poteva arrivare fino alla dissoluzione, per i peccati più legati alla fisicità, come la lussuria: Maria Egiziaca espia il meretricio sopravvivendo nel deserto per quarantasette anni con tre pani ${ }^{9}$, così come Maria Maddalena conclude la sua esistenza terrena saziandosi non con alimenti terreni, ma presso i banchetti celesti così che «corporalibus alimentis nullatenus indigebat» ${ }^{10}$.

Tuttavia il pane e il vino sono alla radice stessa del Cristianesimo, del cui culto e della cui liturgia sono componenti basilari. Ancora il pane e il vino sono tra l'altro occasione di due tra i più importanti miracoli di Gesù, la moltiplicazione dei pani nel deserto e la trasformazione dell'acqua in vino alle nozze di Cana, ambedue avvenuti secondo una tradizione medievale nello stesso giorno dell'Epifania, che per questo motivo viene celebrata anche come Phagiphania, la manifestazione della divinità attraverso il cibo. E l'etimologia e il significato ebraico di Betlemme è 'casa del pane', in cui, come ricorda Ambrogio, è appunto nato il pane che discende dal cielo ${ }^{11}$. E ogni martire, come dice uno dei primi testimoni, sant'Ignazio, è come frumento che deve passare attraverso la macina dei denti delle bestie feroci per divenire il pane di $\mathrm{Cristo}^{12}$. Anche l'atteggiamento disponibile di Pietro, dopo le note resistenze, verso le conversioni dei non circoncisi, ovvero il superamento della legge giudaica nella nuova alleanza, è segnato nel testo degli Atti degli apostoli dal cibo, con una visione di una sorta di grande tovaglia, colma di ogni sorta di animali della terra e di uccelli del cielo. Una voce lo invita a mangiarne e alla risposta di Pietro «No davvero o Signore, poiché io non ho mai mangiato nulla di profano o di immondo» la voce mette in chiaro: «Quello che Dio ha purificato, tu non chiamarlo immondo» ${ }^{13}$. E nel vangelo di Marco si commentano delle parole di

9 Cfr. Jacobus de Voragine, Legenda aurea, LIV 30-34, (Stella 2007, 424-425).

10 Cfr. Jacobus de Voragine, Legenda aurea, XCII 133: «Non sentiva assolutamente il bisogno di alimenti corporali» (Stella 2007, 712-713).

11 Ambrosius Mediolanensis, De Iacob et vita beata, CSEL 32, 2 (Schenkl 1897), II, VII, PL 14, col. 627: «Vere domus panis, quae domus Christi est, qui nobis panis salutaris advenit e coelo».

12 «Frumentum Christi sum, bestiarum dentibus molar ut panis mundus efficiar», citato da Jer. De vir. Ill. XVI 18.

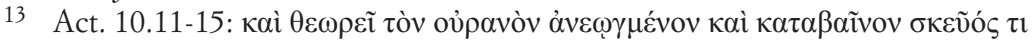

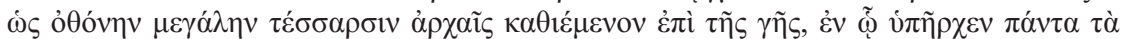

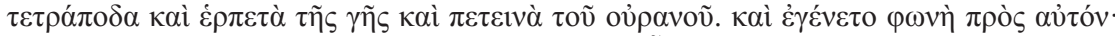

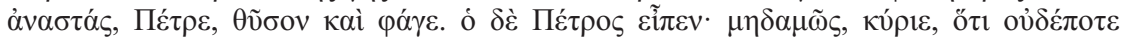


Gesù su ciò che entra ed esce dall'uomo dicendo che così «dichiarava puri tutti gli alimenti» ${ }^{14}$. All'opposto, la disponibilità di Gesù a condividere pasti con pubblicani e peccatori gli vale l'epiteto di «mangione» e di «beone» da parte di scribi e farisei ${ }^{15}$.

Come ha recentemente ricordato Massimo Montanari, la considerazione di cui godono pane e vino è nata nel Mediterraneo orientale ben prima dell'avvento del Cristinesimo, che ne ereditò la tradizione cultura$\mathrm{le}^{16}$ : già nell'Epopea di Gilgamesh il passaggio alla civiltà da parte degli uomini che vivevano allo stato selvatico è segnato dal non limitarsi al consumare i cibi naturalmente disponibili, come acqua, latte, erbe e frutti selvatici, ma quando comincia a consumare pane e bevande fermentate. Allo stesso modo, in Omero la selvaggia incoltura di Polifemo è sottolineata dal fatto che «non somigliava a uomo mangiatore di pane» ${ }^{17}$. Infatti

Il segreto del lievito, da cui pane e vino prendono forma e sostanza, è quello dei microrganismi che modificano la materia e che condurrebbero rapidamente a putrefazione, se l'intervento dell'uomo non cambiasse segno all'azione della natura, trasformando la decomposizione in creazione, il negativo in positivo. ${ }^{18}$

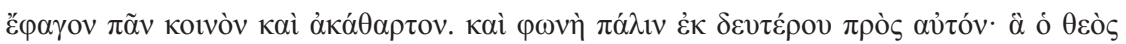

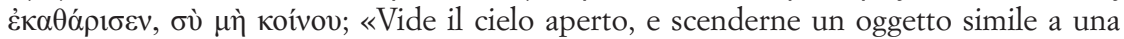
gran tovaglia, che, tenuta per i quattro angoli, veniva calata a terra. In essa c'era ogni sorta di quadrupedi, rettili della terra e uccelli del cielo. E una voce gli disse: 'Àlzati, Pietro; ammazza e mangia'. Ma Pietro rispose: 'No assolutamente, Signore, perché io non ho mai mangiato nulla di impuro e di contaminato'. E la voce parlò una seconda volta: 'Le cose che Dio ha purificate, non farle tu impure'».

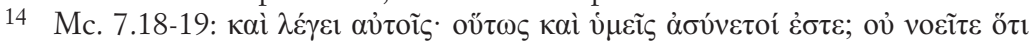

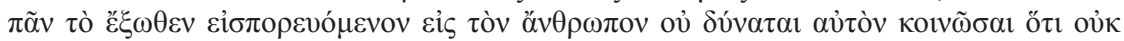

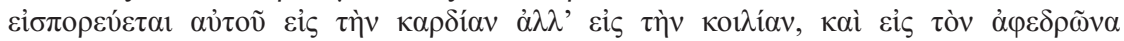

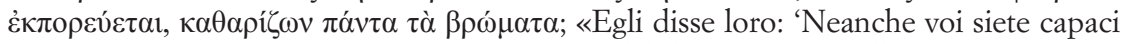
di comprendere? Non capite che tutto ciò che dal di fuori entra nell'uomo non lo può contaminare, perché non gli entra nel cuore ma nel ventre e se ne va nella latrina?'. Così dicendo, dichiarava puri tutti i cibi».

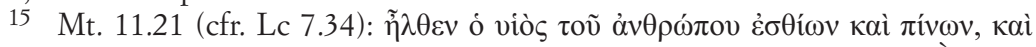

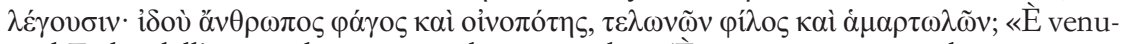
to il Figlio dell'uomo che mangia e beve, e si dice: 'E un mangione e un beone, amico di pubblicani e di peccatori!'. Va ricordato che anche il pubblicano Matteo/Levi festeggia la propria conversione con un grande banchetto a cui Gesù partecipa suscitando ulteriore scandalo (Cfr. Mt. 9.9-13, Lc. 5.27-32, Mc. 2.13-17).

16 Montanari 2015, 27-8.

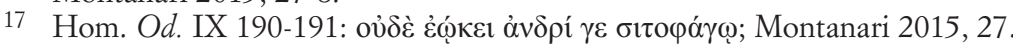

18 Montanari 2015, 27. 
D'altra parte, il peccato originale è stato pur sempre commesso mordendo un frutto ${ }^{19}$, Esaù ha barattato il suo diritto di progenitura per un piatto di lenticchie ${ }^{20}$ e Gesù stesso viene tentato nel deserto dal diavolo che lo invita a tramutare in pani le pietre per placare la sua fame ${ }^{21}$.

Appunto questa ambiguità del cibo, parte imprescindibile del culto cristiano, occasione di miracoli, ma insieme di tentazioni diaboliche è caratteristica anche della letteratura agiografica, proprio perché ogni santo è in primis un imitatore di Cristo. Cibo come tentazione dunque, ma pure cibo come componente necessario della vita umana, e quindi occasione di miracoli.

\subsection{Tra bisogno e piacere. Il cibo, il pane e il vino nei racconti agiografici anteriori al XII secolo}

Come è stato più volte osservato, il cibo è appunto un bisogno ineludibile dell'uomo e il vizio che gli è connesso, la gola, è classificato come vizio carnale: gustare cibi è, oltre che naturale, inevitabile per chi voglia sopravvivere ${ }^{22}$. Perlomeno fino al XII secolo, quando nella religiosità e nel sentire comune inizierà ad apparire una nuova idea di corpo, il concetto di peccato - e dunque buona parte dei temi della letteratura agiografica, che questo concetto accolgono e interpretano -, si articola tra il bisogno e il piacere, poiché proprio questo piacere è la porta da cui si insinua il peccato, come aveva chiarito Gerolamo nell'Adversus Jovinianum ${ }^{23}$. La letteratura agiografica per grande parte del Medioevo è dunque improntata sulla disciplina monastica, poiché è la virtù monastica per eccellenza, la discretio, che può guidare al difficile equilibrio tra le esigenze di sostentamento e il cammino ascetico verso la perfezione ${ }^{24}$. D'altra parte per secoli i principali destinatari dei testi agiografici risiedettero nelle mura di un monastero. Con la proposizione di modelli solo ammirabili per il loro rifiuto radicale, si proponeva in realtà uno stile imitabile, fatto di austerità e di rinunce ${ }^{25}$. Questo

19 Gn. 3.1-7.

20 Gn. 25.29-34.

21 Mt. 4.3.

22 Jer. Adv. Iovinian. II 10, PL 23, col. 313. Vecchio 2009, 29-30.

23 Jer. Adv. Iovinian. II 8, PL 23, col. 310.

24 Casagrande-Vecchio 2000, 130.

25 La terminologia «ammirabile/imitabile» fa ovviamente riferimento a Vauchez 2000, 57-68. 
paradigma interpretabile travalicherà anche la soglia del XII secolo, influenzando i testi agiografici riguardanti le origini dei nuovi ordini religiosi, come la Vita (o le Vitae) di Bernardo di Chiaravalle, dove i primi cisterciensi sotto la sua guida ddurante la fondazione di Clairvaux si nutrono con alimenti a base di faggio e pane di farine miste d'orzo, miglio e veccia ${ }^{26}$ e dove lo stesso Bernardo non è in grado di distinguere i sapori di cibi elementari ${ }^{27}$, interpretando così nella Francia del XII secolo le figure ascetiche che ai cibi rinunciavano radicalmente: ma in questo nuovo contesto non si tratta più di astenersi dall'ingerire, ma di astenersi dall'assaporare e dal rifuggere dalla curiositas $^{28}$.

Nei testi agiografici anteriori al XII secolo il cibo è dunque occasione di tentazione e di peccato e i miracoli sono per lo più legati all'adesione a (o, all'opposto, alla trasgressione di) un ascetismo di tipo monastico oppure, per i laici, al semplice soddisfacimento del bisogno alimentare essenziale per la sopravvivenza. Altri miracoli introducono il meraviglioso nella vita reale, mostrando il soccorso del santo agli affamati, ma gli alimenti sono sempre, salvo poche eccezioni, assai umili. Si tratta quasi sempre di pane, il cibo per antonomasia, come riportano le etimologie medievali: secondo Uguccione da Pisa panis è legato a pan (tutto), perché si accompagna a ogni cibo e ogni animale se ne nutre ${ }^{29}$. Nei testi agiografici compare nelle sue varie qualita ${ }^{30}$, a partire dal pane similagius, il miglior pane di frumento, però citato in esempi negativi, come quello relativo a un gabelliere nella Vita di san Giovanni elemosinario che prima della sua misericordiosa conversione lo tira per sbaglio (cercava in realtà una pietra) a un povero per scacciarlo ${ }^{31}$. Nel caso compaia in esempi positivi, è prerogativa celeste di Cristo stesso che lo offre

26 Vitae Bernardi, in Boninus Mombritius, Sanctuarium seu Vitae Sanctorum, Paris, Fontemoing, 1910 (ried. Hildesheim-New York, Olms, 1978), I 183; Alanus Autissiodorensis, Vita Bernardi, PL 185, coll. 469-524, VI, col. 480.

27 Vitae Bernardi, in Boninus Mombritius, Sanctuarium seu Vitae Sanctorum, Paris, Fontemoing, 1910 (ried. Hildesheim-New York, Olms, 1978), I 182, cfr. Guillelmus Sancto Theodorici, Vita Bernardi, I, VII, PL 185, col. 247.

28 Thomae de Aquino, Quaestiones disputatae de malo (1266-1272), q. 14, a. 3 , ad 4, in Sancti Thomae De Aquino Opera omnia iussu Leonis XIII P. M., Roma-Paris, 1982 (Fiorentino 2001); Vecchio 2009, 37 s.

29 Uguccione da Pisa, Derivationes: «Item a pan hic panis -nis, quia cum omni cibo apponatur vel quia omne animal eum appetat» (Cecchini 2004, 893).

30 Sul pane nei testi medievali anteriori al XIII secolo si veda Bautier 1984, 33-65.

31 Vita sancti Johannis eleemosynarii XXI, PL 73, col. 356. 
ai martiri prigionieri nel suo nome, come a santa Eugenia ${ }^{32}$, oppure al presbitero Santolo, che voleva costruire una chiesa in onore di san Lorenzo $^{33}$. All'altro estremo della scala, c'è invece il pane d'orzo, il peggiore, di converso citato in esempi positivi, come il cibo dei santi in umile penitenza. Uno dei cattivi per antonomasia, Giuliano l'Apostata, si offende quando san Basilio gli offre del pane d'orzo e contraccambia con del fieno, accompagnandolo con le parole, «mi hai offerto il cibo che si dà agli animali, prenditi quello che ci hai dato» ${ }^{34}$. C'è da dire che anche Costantino l'Africano, trattando di dietetica, lo considerava assai poco nutriente ${ }^{35}$. In mezzo il pane subcinericius, senza lievito e, appunto, cotto sotto la cenere, un pane comune, di penitenza, ma anche adatto ad essere portato dai pellegrini nelle loro bisacce, come mostrano alcuni miracoli avvenuti sulla via per Santiago di Compostela ${ }^{36}$. Questo

32 Vita sanctae Eugeniae virginis et martiris XXIX, PL 73, col. 620.

33 Gregorio Magno, Dialogi III 37; cfr. Pricoco-Simonetti 2006, 150-151.

34 Jacobus de Voragine, Legenda aurea, XXX 104-128, in part. par. 104-109: «Sanctus Basilius eidem [scil. Iuliano imperatori] occurrit et panes ordeaceos pro munere ei misit. At Iulianus indignatus eos recipere contempsit et pro panibus sibi fenum misit dicens: 'Pabulum irrationabilium animalium nobis obtulisti, recipe quod misisti'. Respondit Basilius: 'Nos quidem quod comedimus tibi misimus. Tu uero dedisti nobis unde bestias tuas nutris'»; «San Basilio gli andò incontro [scil. a Giuliano l'Apostata] e gli offrì in dono dei pani d'orzo. Ma Giuliano indignato si rifiutò di accettarli e per ricambiare il pane offrì del fieno, dicendo: 'Ci hai offerto il cibo che si dà agli animali, prenditi quello che ci hai dato'. Basilio rispose: 'Noi ti abbiamo appunto mandato quello che mangiamo. Tu invece ci hai dato quello che dai da mangiare ai tuoi animali'» (Stella 2007).

35 Liber Pantegni Ysaac Israelite... quem Constantinus Africanus... sibi vindicavit, ed. Lyon 1515, V 19, f. 21 rb: «panis ordeaceus... minus nutritivus», cit. in Bautier 1984, 37-38.

36 Cfr. ad es. il Mir. 14 del Liber Sancti Jacobi Codex Calixtinus citato da Jacobus de Voragine, Legenda aurea, XCV 167-169: «Cum cuidam uiro de Viziliaco, ut ait Calixtus papa, ad sanctum Iacobum properanti pecunia defecisset et ille mendicare erubesceret, sub quadam requiescens arbore sompniabat quod sanctus Iacobus eum pascebat. Euigilans igitur subcinericium panem ad caput repperit, de quo XV diebus uixit quousque ad propria remeauit. Singulis enim diebus sufficienter bis de ipso comedens sequenti die ipsum integrum in sacculo inueniebat»; «Papa Callisto dice che a un uomo di Vézelay in viaggio per Santiago erano finiti i soldi, ma si vergognava a mendicare. Mentre riposava sotto un albero sognò san Giacomo che gli dava da mangiare. Una volta sveglio trovò vicino alla testa del pane cotto sotto la cenere, del quale visse per quindici giorni, finché ritornò a casa sua.Ogni giorno infatti ne mangiava due volte a sufficienza e il giorno dopo lo ritrovava intero nella propria borsa» (Stella 2007, 736-737). 
pane, per come veniva preparato, aveva anche un ovvio significato penitenziale e un valore simbolico, poiché rimandava a Gesù Cristo, simboleggiando sia la sua carne umana soggetta alla morte, sia il candore della sua anima, ambedue unite nella sua persona ${ }^{37}$.

Per quanto riguarda il vino, pur nel comune e ovvio aborrimento di ogni ebrietas, ci sono differenti sfumature tra i testi che in un modo o nell'altro risentono dell'influenza greca orientale, più ascetici e rigorosi, e quelli cresciuti nell'ambito occidentale, più tollerantii ${ }^{38}$. Nel monachesimo occidentale il vino è previsto, come dice san Benedetto: «Per quanto leggiamo che il vino non è assolutamente per i monaci, pure, siccome oggi non è possibile persuaderne i monaci, acconsentiamo che almeno non si beva fino alla sazietà, ma con moderazione perché 'il vino fa apostatare anche i saggi'» ${ }^{39}$. Tra le ragioni di questa accondiscendenza, lasciando da parte l'uso liturgico del vino, vi è un concorso tra le motivazioni di natura igienico sanitaria - lo stato di fonti e pozzi soprattutto in regioni dove la pioggia non era così frequente - e anche un atteggiamento culturale di antica tradizione che vedeva il vino come la bevanda

37 Si trattava di un argomento utilizzato nella predicazione, poiché era un efficace rimando al mondo quotidiano degli ascoltatori. Cfr. Jacobus de Voragine, Sermones de sanctis. Volumen breve, s. 399 Schn.: «Tertio posuit tertium ferculum, scilicet sacramentum charitatis in sui corporis et sanguinis consecratione cum dicitur Gn. XVIII 'Ponamque buccellam panis'. Iste panis fuit formatus in arca uteri virginalis de tribus farinis, scilicet antiqua, nova, et æterna; id est, carne, anima, et deitate vera. Hoc significatum est, cum dicitur III Rg XIX 'Respexit, et ecce ad caput suum subcinericius panis, et vas aquæ'. Nam iste panis est candidus, quantum ad animam puram; et subcinericius quantum ad carnem mortalitati subjectam, et ad caput positus, quia ista duo, scilicet caro et anima, fuerunt in Christo in unitate persone conjuncta»; «In terzo luogo pose il terzo cibo, cioè il sacramento della carità, nella consacrazione del suo corpo e del suo sangue, laddove si legge Gn. 18,5 'Permettete che vada a prendere un boccone di pane'. Questo pane fu formato nella madia dell'utero virginale con tre farine, ossia quella antica, quella nuova e quella eterna; cioè con la carne, l'anima e la vera divinità. Questo viene significato laddove si legge in 1 Re 19, 6 'Egli guardò ed ecco che vicino al capo c'era un pane cotto sotto la cenere e una brocca d'acqua'. Infatti questo pane è candido, per quanto riguarda l'anima del pane; e cotto sotto la cenere, per quanto riguarda la carne soggetta alla morte; e posto vicino il capo, poiché queste due cose, cioè la carne e l'anima, furono in Cristo congiunte nell'unità della sua persona» (Maggioni c.d.s.).

38 Per quanto riguarda il vino e la sua considerazione nel Medioevo, si veda, ad esempio, Verdon 2002 e Jaboulet-Vercherre 2014.

39 Benedetto, Regula, 40 in Pricoco 1995, 114-385, 212. Cfr. Verdon 2002, 16-17. 
delle classi sociali più elevate ${ }^{40}$. Inoltre ci sono dei fattori per così dire «locali», legati all'economia e alla produzione locale, ad esempio quelli che hanno fatto sì che la leggenda agiografica di san Remigio di Reims comporti miracoli riguardanti la moltiplicazione del vino ${ }^{41}$.

Per quanto riguarda l'agiografia di origine orientale, a questo proposito, un interessante miracolo è quello riportato nella vita greca di sant'Epifanio, il quale per dissetare alcuni mercanti sperduti nel deserto, non fa scaturire una sorgente miracolosa seguendo uno dei numerosi esempi attestati nelle Scritture e nei testi agiografici, ma trasforma il loro vino in acqua, con un interessante rovesciamento del paradigma evangelico ${ }^{42}$. Nella tradizione delle Vitae Patrum, che hanno un'importante diffusione anche nell'Occidente latino, ricorrono infatti gli esempi di rigoroso ascetismo in cui il santo anacoreta si astiene anche dal vino. Per esempio, un vecchio monaco reagisce davanti a un calice di vino con le parole «Toglietemi di torno questa morte» ${ }^{43}$ e un

40 Cfr. Montanari 2015, 35 ss.

41 Cfr. ad esempio Jean de Mailly, Abbreviatio in gestis sanctorum, XIX 13-21, 82: «Quodam autem tempore cum parochias circuiret, hospitatus est in domo cuiusdam cognate sue. Et dum de deo in uicem loquerentur, famulus domus consuluit domine sue quod caupo totum iam uinum uendiderat, excepto modico quod pro seruando uase retinuerat. Videns ergo beatus Remigius illam erubescentem, cognita causa confortauit eam. Et statim in uiridarium quasi spatiaturus exiuit et ueniens ante hostium cellarii intrauit et si quid haberet uini quesiuit. 'Ecce', inquit famulus, 'modicum quod remansit'. Et ille: 'Claude', inquit, 'hostium et sta ibi donec reuertar ad te'. Et pergens retro dolium fecit crucem super illud. Cumque flexis genibus oraret, statim uinum per bundonem egreditur ita quod per medium cellarium fundebatur. Quod uidens famulus exclamauit et cucurrit ad dominam suam et hoc quod uiderat nuntiauit»; «Un giorno, mentre stava facendo il giro delle sue parrocchie, fu ospitato nella casa di una sua parente. E mentre conversavano riguardo a Dio, un servo di casa fece sapere alla sua padrona che il cantiniere aveva già venduto tutto il vino, eccetto una piccola quantità che aveva tenuto da parte in una brocca. Vedendola allora arrossire, san Remigio ne chiese la ragione e la confortò. E quindi se ne uscì subito in giardino come per fare due passi e arrivato davanti alla porta della cantina entrò e chiese se avesserto un po' di vino. 'Ecco', disse il servo, 'quel poco che è rimasto'. E lui: 'Chiudi la porta e sta' lì finché non tornerò da te'. E andando dietro la botte, vi fece sopra una croce. E mentre pregava inginocchiato, subito il vino uscì dalla spina così che scorse fino in mezzo alla cantina. E il servo vedendo il miracolo corse dalla sua padrona a raccontargli quello che aveva visto» (Maggioni 2013).

42 Testo greco Rapp 1991, par. 14, 64-65; per l'edizione si veda anche Dindorf 1859, xxxi-xxxvi, 3-78. Testo latino in Vita Epiphanii, 6, in Chiesa-Dolbeau 1989, 930. 43 Verba seniorum V 5.54: «Tolle a me istam mortem», PL 73, col. 871. 
altro scappa atterrito fino a mettere a repentaglio la propria vita in una cripta fatiscente ${ }^{44}$. Allo stesso modo l'abate Pafnuzio viene costretto a berne solo quando un ladrone lo minaccia di morte ${ }^{45}$, l'abate Pietro Pionio se ne astiene anche nell'estrema debolezza della sua senescen$\mathrm{za}^{46}$ e Macario rimprovera se stesso come «vecchio malvagio»

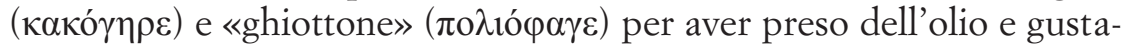
to del vino durante la sua vita ${ }^{47}$. Ancora nella parte costituita dalla raccolta di aforismi chiamata Verba seniorum si dice anche esplicitamente che «Il cibo non è fatto assolutamente per i monaci» ${ }^{48}$.

\subsection{Il latte nella letteratura agiografica}

Il latte, elemento primario per il sostentamento dell'uomo e non presente nella liturgia, ha assunto un valore simbolico ricorrente nella letteratura agiografica soprattutto a partire dai brani scritturali di I Pt. 2.2 «come bambini neonati anelate al latte spirituale e genuino, affinché per mezzo di esso cresciate in vista della salvezza» ( $\dot{\varsigma} \varsigma \dot{\alpha} \rho \tau \imath \gamma \varepsilon \dot{\varepsilon} v v \eta \tau \alpha$

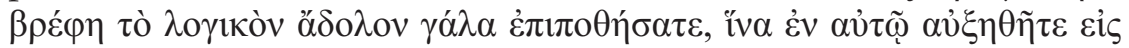

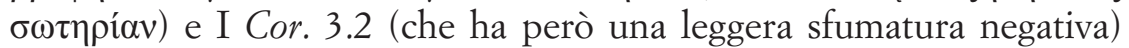
«Ed io, o fratelli, non ho potuto parlare a voi come a degli uomini spirituali, ma come ad esseri di carne, come a infanti in Cristo. Vi ho dato

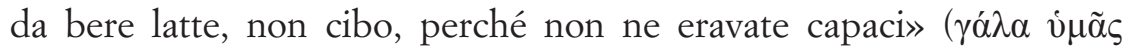

44 Verba seniorum V 5.53, PL 73, col. 871.

45 Verba seniorum III 151, PL 73, col. 791.

46 Verba seniorum V 5.35, PL 73 col. 869.

47 Palladius, Historia Lausiaca, 18.26. Va detto che Palladio stesso nel Prologo (9, ibidem, 10-3) mette pesantemente in guardia contro un ascetismo solo formale e un

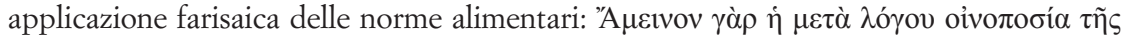

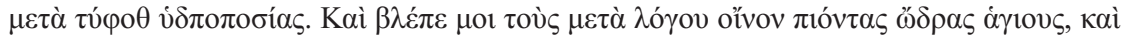

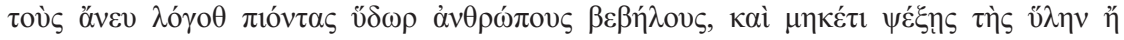

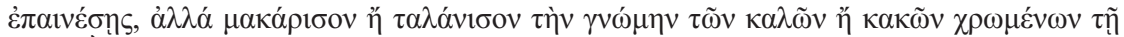
v̋ $\lambda \eta ̣$; «E preferibile bere il vino con criterio al bere l'acqua con vanità. Considera uomini santi quelli che bevono il vino seguendo la ragione e uomini corrotti quelli che bevono l'acqua irrazionalmente, e non biasimare e lodare la materia, ma esalta o deplora l'intenzione di coloro che fanno un buono o cattivo uso della materia» (Bartelink 1974, 94).

48 Verba seniorum V 5.35, PL 73 col. 869: «Narraverunt quidam abbati Pastori de quodam monacho, qui non bibebat vinum. Et dixit eis: 'Qui vinum monachorum omnino non est'»; «Riferirono all'abate Pastore di un monaco che non beveva vino. E lui disse loro che 'Il vino non è assolutamente fatto per i monaci'». 


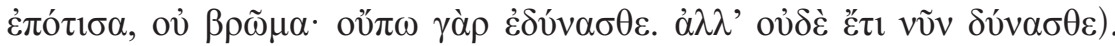
L'immagine venne quindi ripresa nella letteratura patristica. Clemente di Alessandria nel suo Pedagogo propone l'etimologia di $\mu \alpha \sigma \tau \varepsilon v \tilde{\sigma \alpha}$ collegandolo con l'immagine delle mammelle (in greco

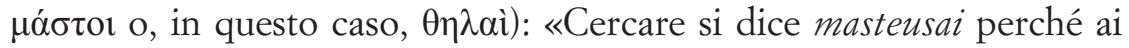
bambini che cercano il Logos i seni generosi del Padre procurano il latte $»^{49}$. Nel trattato l'immagine ricorre più volte, ad esempio: «Noi ci rifugiamo nel seno del Padre, che fa dimenticare i dolori, nel Logos. Lui solo, come una cosa naturale, dona a noi piccoli il latte dell'amore e beati coloro che sono nutriti da questo seno» ${ }^{50}$. Più o meno contemporaneamente, Ireneo riprende il passo paolino riaffermando che Cristo, essendo il pane perfetto del Padre, ci si offre come latte attraverso la mammella della sua carne ${ }^{51}$. Nella letteratura esegetica dei primi secoli dell'era cristiana l'immagine del latte veniva dunque impiegata per indicare come un alimento propedeutico associato alla catechesi di base e come simbolo della predicazione e dell'insegnamento.

Nella tradizione agiografica alto medievale l'immagine del latte è più spesso associata al martirio invece che al nutrimento materiale. Questo utilizzo del tema si ricollegava all'origine emogena del latte che, secondo la medicina medievale, era appunto sangue cotto $^{52}$. In questo caso il sangue del martire viene cotto dalla carità e dalla passione diventando nutrimento

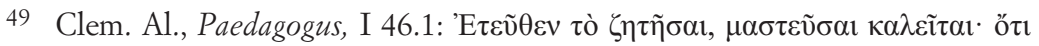

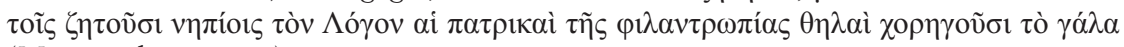
(Marcovich 2002, 29).

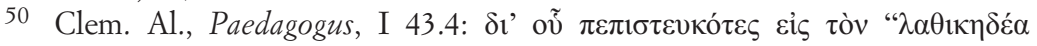

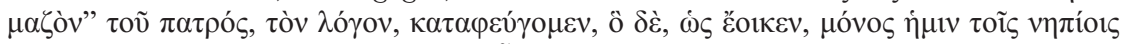

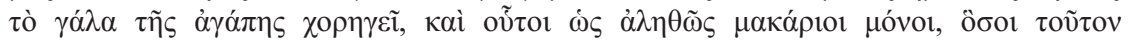

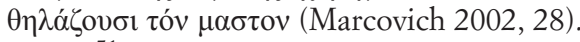

51 Irenaeus Lugdunensis, Contra Hereses, IV 38.1, PG 7, col. 1196: «Et propter hoc, quasi infantibus, ille qui erat panis perfectus Patris lac nobis semetipsum praestavit, quod erat secundum hominem eius adventus: ut quasi a mamilla carnis eius enutriti, et per talem lactationem assueti manducare et bibere Verbum Dei, et eum qui est immortalitatis panis, qui est Spiritus Patris, in nobis ipsis continere possimus»; «E per questo, come a dei bambini, colui che era il pane perfetto del Padre offrì a noi se stesso come latte, ciò che era in quanto uomo il suo avvento, così che quasi nutriti dalla mammella della sua carne e abituati da un allattamento di questa natura a mangiare e a bere il Verbo di Dio, possiamo assumere in noi stessi colui che è il pane dell'immortalità, che è lo Spirito del Padre».

52 La teoria dell'origine emogena del latte risale ad Arist. GA IV 8 777a4-11 (a questo proposito cfr. Coles 1995, 48-88, part. 54). Così anche Alberto Magno, De Natura 
spirituale (dunque il latte scorre durante il martirio di Paolo ${ }^{53}$, di Caterina ${ }^{54}$, delle matrone di san Biagio ${ }^{55}$ e di Cristina $\left.{ }^{56}\right)$. È qui sottesa l'immagine del latte come sangue dealbato, non solo nutriente, ma anche purificato e purificante, derivata dall'interpretazione di $A p \cdot 7.14^{57}$. In questi casi non si tratta di ingestione: vi sono soggetti che profondono latte, ma non vi sono beneficiari espliciti dell'allattamento. L'allattamento vero e proprio ricompare in racconti agiografici collegati a Bernardo

Animalium, 1. XVIII, tr. 2, c. 7, ed. Stadler 1920, 1235-1237. Per Iacopo da Varazze, era addirittura cotto due volte; cfr. Jacobus de Voragine, Sermones Quadragesimales, f. IV, II hebd., s. II: «Omnis autem alius sanguis habet inficere; solus vero sanguis Christi habet dealbare et ideo habet naturam lactis, nec mirum: si enim lac est sanguis bis coctus, scilicet in venis et in mamillis, et in virtute illius duplicis decoctionis convertitur in albedinem, multo fortius sanguis Christi dealbare habebit, qui non tantum bis, sed ter fuit excoctus, scilicet igne amoris [...] igne passionis [...] igne doloris»; «Ogni altro sangue infatti macchia; solo il sangue di Cristo può rendere candidi e perciò ha la natura del latte e questo non deve meravigliare: se infatti il latte è sangue cotto due volte, cioè nelle vene e nelle mammelle, e in virtù di questa duplice decozione acquista la sua albedine, molto più fortemente potrà rendere candidi il sangue di Cristo, che non solo due, ma tre volte fu cotto, cioè dal fuoco dell'amore» (Maggioni 2005, 154).

53 Cfr. Ordericus Vitalis, Historia ecclesiastica, II, VIII, PL 188, col. 137: «De eius autem uulnere unda lactis usque in uestimento militis exiuit et postea sanguis effluxit,in aere lux immensa emicuit, de corpore odor suauissimus emanauit»; «Dalla ferita sprizzò un fiotto di latte che arrivò fino agli abiti del soldato; in seguito ne uscì sangue, in cielo sfavillò una luce immensa, dal suo corpo emanò un profumo soavissimo».

54 Passio Catherinae, in Bonius Mombritius, Sanctuarium seu Vitae Sanctorum, Paris, Fontemoing, 1910 (ried. Hildesheim-New York, Olms, 1978), 287: «Veniens autem spiculator amputavit cervicem eius, et pro sanguine lac emanavit in terra»; «Venne allora il boia e le tagliò la testa; e al posto del sangue latte fluì per terra».

55 Passio Blasii, in Bonius Mombritius, Sanctuarium seu Vitae Sanctorum, Paris, Fontemoing, 1910 (ried. Hildesheim-New York, Olms, 1978), 152: «Mox autem iussit praeses eas suspendi et laniari carnes earum cum pectinibus illis ferreis. Videbant autem milites illi quod pro sanguine lac carnes earum stilabant»; «Subito il prefetto ordinò di legarle e di lacerare le loro carni con i pettini di ferro. E i soldati videro allora che le loro carni stillavano latte al posto di sangue».

56 Passio Christinae, in Bonius Mombritius, Sanctuarium seu Vitae Sanctorum, Paris, Fontemoing, 1910 (ried. Hildesheim-New York, Olms, 1978), 363: «Ille ira commotus iussit incidi mamillas eius, quibus abscissis pro sanguine lac fluebat»; «Quello fuori di sé dall'ira ordinò di tagliarle i seni; e quando le furono asportati ne fluì latte anziché sangue».

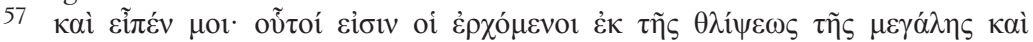

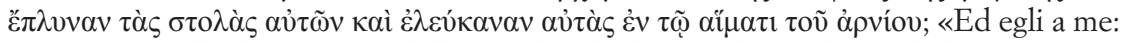
'Essi sono quelli che vengono dalla grande tribolazione: hanno lavato le loro vesti rendendole candide nel sangue dell'Agnello'». 
di Chiaravalle, di cui si inizia a narrare all'inizio del XIV secolo del suo allattamento miracoloso da parte della Vergine ${ }^{58}$. La cosa è particolarmente significativa perché nelle Vitae che raccontano l'infanzia di Bernardo è messo in risalto come la madre si fosse sempre rifiutata di dare a balia i suoi figli «quasi che con il latte materno in qualche modo infondesse in loro la buona indole materna $\gg^{59}$, un racconto in cui si può ravvisare un'eco della figura agostiniana e del cristianesimo che santa Monica infuse a suo figlio insieme al latte ${ }^{60}$. Fu proprio l'allattamento miracoloso infatti a mettere in condizione san Bernardo di predicare presso il vescovo di Chalons. Qui la tradizione è evidentemente legata al concetto di nutrimento spirituale visto nelle epistole di Pietro e Paolo, ripreso più volte da Bernardo e dai suoi confratelli tanto da far diventare il seno che allatta uno dei temi ricorrenti della spiritualità cisterciense, anche riferito a prelati e a abati ${ }^{61}$.

58 La prima attestazione scritta sembra essere nella raccolta in antico francese Ci nous dit; cfr. Blangez 1979-1986, 705, 7-12.

59 Vitae Bernardi, in Bonius Mombritius, Sanctuarium seu Vitae Sanctorum, Paris, Fontemoing, 1910 (ried. Hildesheim-New York, Olms, 1978), I 176: «quasi cum lacte materno materni quodam modo boni infundens eis naturam».

60 Augustinus Aurelius, Confessionum libri tredecim, CCSL 27, ed. L. Verheijen, Turnhout 1981, III, 4: «et hoc solum me in tanta flagrantia refrangebat, quod nomen Christi non erat ibi, quoniam hoc nomen secundum misericordiam tuam, domine, hoc nomen salvatoris mei, fili tui, in ipso adhuc lacte matris tenerum cor meum pie biberat et alte retinebat»; «E questo solo mi abbatteva nel mio entusiasmo così grande, che non ci fosse lì il nome di Cristo, poiché questo nome, per la Tua misericordia Signore, questo nome del mio Salvatore, di Tuo Figlio, il mio cuore ancora tenero aveva piamente bevuto con lo stesso latte materno e l'aveva conservato intimamente».

61 A questo proposito si veda Bynum 1982, 115-125. Ad esempio Bernardo scrive a suo nipote Roberto (Epistolae I 10, ed. Leclerq-Rochais, v. VII, 8): «Nutrivi deinde lacte, quod solum adhuc parvuus capere poteras»; «Ti ho nutrito con latte, quando ancora bambino potevi prendere solo quello». Oppure nel sermone 10 Super Cantica Canticorum, rivolgendosi ai coloro che hanno la responsabilità della direzione delle anime li esorta ad allattare coloro che sono loro affidati (in Bernardi opera, ed. LeclercqTalbot-Rochais 1957-1958, I, 50): «Quae mater est, non dissimulat; habet ubera, et non vacua. Gaudere cum gaudentibus, flere cum flentibus novit, nec cessat exprimere de ubere quidem congratulationis lac exhortationis, de ubere vero compassionis lac consolationis»; «Colei che è madre non dissimula: ha i seni, e non sono vuoti. Sa gioire con chi gioisce e piangere con chi piange, e non cessa di spremere dal seno della sua compartecipazione il latte dell'esortazione e dal seno della compassione il latte della consolazione». Grazie a Umberto Eco è divenuto celebre il passo del sermone 31.4 di Gilberto de Hoylandia Sermones in Canticum Salomoni PL 184, col. 163: «Pulchra sunt enim ubera, 
D'altra parte, più o meno contemporaneamente l'allattamento della Vergine è riportato anche in altri racconti esemplari dove il miracolo è il semplice risanamento del corpo, svincolandosi dall'idea della trasmissione della dottrina e del sapere. Ad esempio, Vincent de Beauvais nello Speculum Historiale racconta di un chierico che proprio venendo allattato dalla Vergine, a cui era molto devoto, viene risanato da una malattia per il cui dolore si era lacerato le labbra e strappato la lingua (tanto è vero che la preghiera alla Vergine viene pronunciata per lui dal suo angelo custode ${ }^{62}$.

L'allattamento certo continua ad indicare la maternità spirituale e per questo in tale ambito sono visibili rovesciamenti delle funzioni naturali, per cui è il maschio che allatta la femmina: è questo il caso di una visione di santa Chiara che si vede allattata da san Francesco, secondo una testimonianza di suor Filippa al processo di canonizzazione che però rimase esclusa dalla leggenda ufficiale ${ }^{63}$. Nella cappella Bardi in

quae paululum supereminent, et tument modice; nec elata nimis, nec tamen aequata carni reliquae; quasi repressa, sed non depressa; leniter restricta, non fluitantia licenter. Hoc quidem exemplo qui verba bona, verba spiritualia debet proferre, mulierum imitetur et studia, et industriam. Castigato utatur sermone, verborum non fluitent ubera, non sine lege ferantur; non pectus et quoddam secretarium mentis magis occupent, quam ornent; non habeant plus materiae quam gratiae; non plus carnis quam lactis»; «Belli sono infatti i seni che si elevano di poco e sono misuratamente tumidi, trattenuti, ma non compressi, legati dolcemente e non liberi di ondeggiare [fin qui trad. di Eco 2009, 54 5]. Si faccia uso di un'oratoria castigata, non ondeggino i seni delle parole, non si muovano senza ordine. Non ingombrino il petto e quella certa parte più riposta della mente più di quanto li adornino. Non abbiano più materia che grazia, più carne che latte».

62 Vincentius Bellovacensis, Speculum Historiale, VIII 84, cfr. Balthazar Bellerus 1624, 251-252. Questa la descrizione della guarigione: «Hec cum ille in auribus tamen egri declamaret et in eius oculis deploraret, apparuit utrique mater domini et quasi satisfaciens de negligentia in servulum suum de qua insimulabatur ad lectulum egri propius accedit. Extractaque ubera visa est protinus immittere in os egri et sancti lactis sui rore infuso subito lingue integritatem, et labiis antiquum decorem, et in toto eius corpore pristinam restauravit sanitatem»; «E mentre egli [scil. l'angelo custode] diceva queste all'orecchio dell'ammalato, apparve ad entrambi la Madre di Dio e quasi ponendo rimedio a quella negligenza verso il suo servo che era stata insinuata, si avvicinò al letto dell'ammalato. E sembrò scoprirsi i seni e offrirli alla bocca dell'ammalato. E subito con l'infusione del suo latte rugiadoso subito gli restituì l'integrità della lingua, l'antica bellezza delle labbra e la sanità originaria nell'intero corpo». L'esempio è stato ripreso anche da Filippo da Ferrara, Liber de introductione loquendi, I 27, cfr. Vecchio 1998, 160.

63 Processo di canonizzazione, Terza Testimonia (Suor Philippa de Gislerio), in Menestò-Brufani-Cremascoli 1995, 2467 e ss. Cfr. Lionetti 1988, 90-94 e Bartoli 1980, 449-472. 
Santa Croce Francesco è d'altronde raffigurato sicut mater, tenendo in grembo un lebbroso, in una posa molto simile alle rappresentazioni della Virgo lactans ${ }^{64}$. A questo proposito va tra l'altro ricordato come il termine mater sia stato estremamente importante nel francescanesimo per definire il minister che nutre e cura i confratelli. Francesco nella nota lettera autografa inviata a padre Leone e conservata nella Cappella delle reliquie della cattedrale di Spoleto, si presenta sicut mater («ita dico tibi fili mei sicut mater», «Così dico a te, figlio mio, come una madre» $\left.{ }^{65}\right)$ e lui stesso all'aggravarsi della malattia sceglie frate Elia «come madre per se stesso e padre per gli altri frati» $»^{66}$. E in area irlandese nel tardo Medioevo si incontra una tipologia di santi maschi e allattatori la cui azione si è allontanata dalla valenza simbolica ${ }^{67}$, in un contesto caratterizzato non solo da una persistenza nel folklore del tema antropologico della «covata rituale» e da pratiche d'adozione, attestate nella antica cultura gaelica ${ }^{68}$, per mezzo di una simulazione dell'allattamento e di una sottomissione per mezzo della suzione del seno, ma anche da un approccio particolare alle tematiche corporali ${ }^{69}$. Il tema iniziale è pur sempre l'iniziazione e la trasmissione del sapere, ma progressivamente si affermano leggende in cui il latte è solo un nutrimento di origine corporale (per quanto miracolosa la sua origine) che permette ai santi di sovvenire bambini abbandonati destinati alla morte per fame.

Nel Libro di Lismore, una compilazione in antico irlandese dell'inizio XV secolo, ma dipendente da raccolte agiografiche precedenti

64 La tavola, nella cappella omonima di Santa Croce a Firenze, è databile nel 1243 circa, ed è ispirata alla Vita prima di Tommaso da Celano e al Testamento di Francesco d'Assisi. Si veda Cassio-Messa 2015, 14 e fig. 1.

65 A questo proposito si veda Dalarun 2009, 19-51.

66 Thomae de Celano, Vita prima beati Francisci, IV 98, in Menestò-BrufaniCremascoli 1995: «quem loco matris elegerat sibi, et aliorum fratrum fecerat patrem».

67 Sui santi irlandesi e l'allattamento, cfr. Gricourt 1957a, 249-257 e Gricourt 1957b, 187-194.

68 Così Lionetti 1988, 102. Per 'covata rituale' si intende la sostituzione della madre da parte del padre dopo il parto, una ritualità diffusa e studiata dagli antropologi. Sulla sua persistenza in ambito irlandese (e più generalmente insulare) si veda Cross 1969, T 583.1, Delaisi de Parseval 1981, 16-7.

69 Si veda, ad esempio, il viaggio nell'Aldilà in corpore del cavaliere Owein nel Purgatorio di san Patrizio e l'intera tradizione di pellegrinaggio che vi si riallaccia. Si tratta di documenti storici letterari dove è evidente un'influenza di Echtrae e Immram generi tradizionali della cultura irlandese. Cfr. Maggioni-Taviani-Tinti c.d.s. e, in generale Dumville 1976, 82-83. 
purtroppo oggi perdute, si racconta infatti di Findchua che salvò un bambino destinato morte certa, offrendogli il proprio seno destro stillante latte. Si trattava del piccolo figlio di Aifé, la moglie meno favorita di Nuada il Saggio re di Leinster, che era infatti bigamo e le preferiva Anmet, figlia di Colman, figlio del potente Crimthann di Hui Cennselaig. In questo modo il bambino crebbe nutrito meglio che se fosse stato allattato da nove nutrici ${ }^{70}$.

Ancora, secondo un'altra leggenda agiografica irlandese ${ }^{71}$ Colmán di Lann Ela allattò i gemelli Ultan e Baithin, concepiti incestuosamente e ripudiati, offrendo loro un seno che dispensava latte e uno miele ${ }^{72}$.

In questa stessa direzione in Italia si svilupperà più tardi la leggenda agiografica di san Mama, o san Mamas, o san Mamette, o san Mammano. Originario della Cappadocia, il culto si sviluppò in Occidente soprattutto in seguito al saccheggio di Costantinopoli del 1204 e alla traslazione delle reliquie. La sua leggenda agiografica è connessa alla pastorizia e alla produzione di formaggi e rieccheggia in

70 Sulla storia del manoscritto cfr. la prefazione dell'editore del testo, Stokes 1890, 84-98. Traduzione inglese alle pagine 231-246. Cfr. 237: «The damsel brings forth a boy at an early hour on the morrow, and he is taken from her to Findchua to be baptized. Thereafter the boy is baptized and (the name) Finntan is given to him, even Finntan son of Old Nuada the Sage, son of Bresal the Speckled, son of Fiacha Fobrecc. The boy is reared by Findchua, who gives him his right breast, and milk, grew therein, and his mother is warned to go into her own country. That boy throve as he would not have thriven with his own mother if he had nine wet-nurses under him». Sulla leggenda di san Findchua, cfr. Lionetti 1988, 21-22.

71 Il testo è stato raccolto tra il 1620 e il 1635 da M. O'Clery, un terziario francescano, su incarico dei suoi superiori che, all'Irish College a Lovanio intendevano raccogliere una collezione di antichità irlandesi. Il frutto della ricerca è conservato nei mss. 2324-40 della Bibliothèque Royale de Belgique.

72 Testo gaelico pubblicato da Plummer 1922, I, 168-182. Traduzione inglese ibidem, II, 162-176 (cfr. 167-8: «Said Columcille: 'Úanach, the sister of thy mother, and my own sister, has borne two sons to Maeluma son of Baetan [...]; and I baptized them, and I named one of them Ultan, and the other Baithin. And I would rather that they did not live, but that I could compass their destruction without shame to myself, for their father and mother are children of a brother and sister [NdE: 'Rectius: children of two brothers', says a marginal note, quite correcly]. And I would fain have thy advice as to them'. 'My advice is soon given', said Colman, 'give them to me to nourish and to foster. And let us make a covenant respecting them, for I have two paps such as no saint ever had before, a pap with milk, and a pap with honey, and these I will give to them [to suck]'». Per leggende simili, si veda anche Mac Néill 1921, 54. 
molti punti il mito di Orfeo: fuggito dalle persecuzioni cittadine, Mama predicava il vangelo alle fiere nei boschi, che si radunavano attorno a una fonte fatta da lui scaturire mentre le cerve gli si offrivano spontaneamente per la mungitura. A sua volta Mama faceva pervenire i suoi formaggi ai cristiani incarcerati. La connessione tra il santo e il latte è naturalmente sottesa al suo nome, che gli fu dato poiché chiamava la sua nutrice alla latina Mama ${ }^{73}$. Il culto di san Mama si legò quindi naturalmente, oltre alla protezione dalle fiere selvatiche, ai problemi dell'allattamento e iniziò a essere invocato dalle nutrici la cui secrezione lattea era insufficiente o che soffrivano di mastiti e di ragadi ${ }^{74}$. Ne conseguì quindi uno sviluppo anche della figura agiografica: un giorno che il santo camminava su un sentiero di montagna trovò un bambino abbandonato dai genitori. E Dio gonfiò di latte il seno di san Mamma, che lo potè quindi offrire al bambino sfamandolo ${ }^{75}$. All'opposto, la secrezione miracolosa punisce un incredulo che con i seni gonfi di latte e dolenti in cerca di sollievo si vede costretto a cercare un bambino da allattare ${ }^{76}$.

\subsection{Il cibo nei racconti agiografici posteriori al XII secolo}

Nel XII secolo appunto, oltre a farsi strada una diversa concezione di corpo, appare anche un nuovo concetto di piacere, che viene considerato, da Abelardo in poi, come un moto naturale, ineliminabile, connesso strettamente alla nutrizione e dunque neutro dal punto di vista etico ${ }^{77}$ e in questo caso, come scrive Tommaso, il peccato è quello dell'intemperanza, allo trasgredire in modo per così dire quantitativo alla moderazione aristotelica e alle necessità naturali ${ }^{78}$. Per quanto

73 Cignitti, v. Mama di Cesarea, in BB. SS. VIII, col. 591-611, partic. col. 601. In ambito Occidentale e neolatino è più che ovvia l'associazione con mamilla, mamma, mammella eccetera.

74 Lionetti 1988, 15-16.

75 Narrazione raccolta da Bastanzi 1988, 52. Citato da Lionetti 1988, 14.

76 Nardo Cibele 1887, 326 ss. Citato da Lionetti 1988, 14.

77 Vecchio 2009, 31.

78 Vecchio 2009, 37, che ricorda anche - citando Thomae de Aquino, Sententia libri Ethicorum (1271-1272), III, 20, in Sancti Thomae de Aquino Opera omnia iussu Leonis XIII P.M., Roma 1969, 184 - come «il riferimento alla gola e non alla lingua, organo deputato al gusto, denuncia la completa irrilevanza del ruolo dei sapori nella dinamica del peccato di gola». 
riguarda il cibo esiste però anche un'altro tipo di piacere, artificiale $\mathrm{o}$, citando Silvana Vecchio, «quantomeno soggettivo», legato alla sfera culturale e conoscitiva: la ricerca di cibi estremamente raffinati, costosi o rari, ma anche frutto di accostamenti inconsueti: questo tipo di piacere è invece il legato il concetto più generale del peccato di curiositas $^{79}$.

La letteratura agiografica è specchio di questo cambiamento culturale e nei leggendari si iniziano ad accogliere delle vere e proprie indicazioni dietetiche, come quelle che accompagnano la tradizione dei digiuni delle Quattro Tempora, per portare a un equilibrio tra le qualità elementari del corpo (caldo freddo secco umido) e tra i suoi fluidi (sangue collera flemma e melanconia) mirando dunque al mantenimento o al recupero della salute, del corpo e dell'anima ${ }^{80}$. La primavera è calda e umida, l'estate calda e secca, l'autunno freddo e secco, l'inverno freddo e umido. Ognuno dei quattro digiuni è quindi per così dire specializzato e funzionale per mantenere un equilibrio non solo spirituale, ma anche fisico, per evitare di cadere nei peccati corrispondenti allo squilibrio.

San Domenico soccorre dunque il malato di scrofola non sanando miracolosamente, ma suggerendo una dieta alimentare. Appare notte-

79 Vecchio 2009, 37, citando sia Thomae de Aquino, Quaestiones disputatae de malo (1266-1272), q. 14, a. 3, ad 4, in Sancti Thomae De Aquino Opera omnia iussu Leonis XIII P. M., Roma-Paris, 1982 (Fiorentino 2001), 265 ss., sia la posizione anticipatrice di Giovanni de la Rochelle nella sua Summa de Vitiis.

80 Cfr. Jacobus de Voragine, Legenda aurea, XXXV 2-7, 18-25: «Fiunt autem hec ieiunia quater in anno secundum quatuor tempora ipsius anni et huius est multiplex ratio. Prima est quoniam uer est calidum et humidum, estas est calida et sicca, autumpnus frigidus et siccus, hiemps frigida et humida. Ieiunamus igitur in uere utnoxium humorem, id est luxuriam, in nobis temperemus; in estate ut noxium calorem, id est auaritiam, in nobis castigemus; in autumpno ut castigemus ariditatem superbie; in hieme ut castigemus frigus infidelitatis et malitie; [...] Quinta ratio est quoniam, ut dicit Iohannes Damascenus, in uere augmentatur sanguis, in estate colera, in autumpno melancolia, in hieme flegma. Ieiunamus igitur in uere ut in nobis debilitetur sanguis concupiscentie et inepte letitie; sanguineus enim est libidinosus et letus. In estate, ut debilitetur colera iracundie et fallacie, colericus enim naturaliter est iracundus et uersutus. In autumpno, ut debilitetur melancolia cupiditatis et tristitie; melancolicus enim est naturaliter cupidus et tristis. In hieme, ut debilitetur flegma hebetudinis et pigritie; nam flegmaticus naturaliter est hebes et piger»; «Questi digiuni avvengono quattro volte all'anno secondo le quattro stagioni dell'anno e le ragioni di questo sono molteplici. La prima è che la primavera è calda e umida, l'estate calda e secca, l'autunno freddo e secco, l'inverno freddo e umido. Quindi digiuniamo in primavera per temperare nel nostro corpo l'umore nocivo, cioè la lussuria; in estate per mitigare in noi il calore nocivo, cioè l'avidità; in 
tempo a una madre che aveva fatto un voto per la guarigione del figlio dalla scrofola: «Donna, conosci queste cose?», e gli nominò quattro tipi di cose: bocca verde, pilatro, lapazio e succo di porro. Lei rispose: «Li conosco», e lui: « $\mathrm{Va}$ ' a prenderle e lavorale col succo di porro e fanne un impiastro da mettere sul collo di tuo figlio, e guarirà» ${ }^{81}$.

Cambia la dieta dei santi e il loro comportamento alimentare viene riportato dagli agiografi che non hanno remore a raccontare della passione di Francesco per il pasticcio di gamberi e per il prezzemolo ${ }^{82}$. O che condiva i cibi con il lardo anche di Quaresima, essendo intollerante all'olio $^{83}$. E vi è anche una serie di racconti che hanno come protagonista fra Ginepro, il cuoco del convento di Assisi ${ }^{84}$. Tra l'altro san Francesco produce miracoli anche con il cibo: i pani da lui benedetti resero la salute a molti malati ${ }^{85}$ e così il vino da lui miracolosamente prodotto ${ }^{86}$.

autunno per frenare l'aridità della superbia; in inverno per castigare il freddo dell'infedeltà e della malizia. [...] La quinta ragione è che, come dice Giovanni Damasceno, che in primavera si accresce il sangue, in estate la collera, in autunno la melanconia, in inverno il flegma. Digiuniamo dunque in primavera per fiaccare il sangue della concupiscenza e del piacere vano; infatti l'uomo sanguigno è lascivo e allegro. In estate per debilitare la collera dell'iracondia e dell'inganno: il collerico infatti è per natura iracondo e furbo. In autunno per fiaccare la melanconia della passionalità e della tristezza. Il melanconico infatti è per natura passionale e triste. In inverno, per fiaccare il flegma dell'apatia e della pigrizia. Infatti il flemmatico è per natura apatico e pigro» (Stella 2007, 424-427).

81 Humbertus de Romanis, Vita Dominici A 61 (cfr. Walz 1935), qui citato da Jacobus de Voragine, Legenda aurea CIX 328-335: «Apud Palatiam in Sicilia mulier quedam pauper filium habebat qui infirmitate quadam scrophularum quas pueri in collo habere consueuerunt plurimum affligebatur. Cumque super hoc nullum posset remedium inuenire uouit deo et beato Dominico quod si liberaretur ipsum in opere ecclesie fratrum que tunc construebatur gratis faceret laborare. Sequenti autem nocte quidam in habitu fratris sibi apparuit eique dixit: 'Mulier, cognoscis has res?', nominans ei quatuor genera rerum, scilicet es uiride, pilatrum, lapatium et succum porri. Que cum diceret: 'Cognosco', ille ait: 'Vade et accipe hec et conficias ea cum succo porri et pone ea super bambace et impone collo filii tui et sanabitur'. Euigilans autem hoc fecit et filius eius liberatus uotum matris impleuit» (Stella 2007, 826).

82 Cassio-Messa 2015, 27.

83 Thomae de Celano, Memoriale, XCIV 131, in Menestò-Brufani-Cremascoli

1995, 715. Citato in Cassio-Messa 2015, 27.

84 Cfr. Petrocchi 1960. Una scelta di racconti è presentata in Cassio-Messa 2015, 72-79.

85 Thomae de Celano, Legenda ad usum chori, in Menestò-Brufani-Cremascoli 1995, 9-10.

86 Bonaventura de Balneoregio, Legenda maior sancti Francisci, V 10, in Menestò-Brufani-Cremascoli 1995. 
Accanto all'umile pane nei racconti agiografici del Basso Medioevo compaiono anche vivande elaborate, come i capponi arrosto e i santi se ne nutrono. San Francesco mangia infatti un cappone arrosto in un exemplum: invitato a pranzo da un fedele che appunto preparò per lui un cappone di sette anni, ne diede una coscia a un mendicante il quale, l'indomani, mentre il santo stava predicando, lo tirò fuori dicendo: «Ecco quale carne si mangia il vostro Francesco, che onorate come un santo! Me l'ha data lui ieri sera!». Ma poiché il pezzo di cappone sembrava a tutti un pesce, tutta la gente prese a insultarlo come se fosse pazzo. Quando se ne accorse, arrossì e chiese perdono; e la carne tornò al suo aspetto originario dopo che l'accusatore tornò in senno ${ }^{87}$. Qui si nota che per la folla e per il mendicante la carne di cappone, contrariamente al pesce, era ancora considerata scandalosa in bocca ad un santo. Infatti il pesce è stato a lungo considerato, in quanto apparentemente esangue, non contaminato e lontano dai tabù alimentari, essendo tra l'altro distante da noi mammiferi per il suo habitat, come i volatili, ugualmente estranei e ugualmente più accettabili nell'alimentazione ${ }^{88}$.

87 Thomae de Celano, Memoriale, II 2.78 ss., qui citato da Jacobus de Voragine, Legenda aurea, CXLV 224-232: «Cum apud Alexandriam Lombardie cum quodam honesto uiro hospitatus fuisset $\mathrm{ab}$ eo rogatur ut propter euangelii obseruantiam de omni apposito manducaret. Cui cum eius deuotioni assentiret, ille accurrens caponem septennem preparat manducandum. Manducantibus illis infidelis quidam amore dei elemosinam petiit. Mox uir dei nomen audiens benedictum membrum sibi transmittit caponis. Reserua infelix datum et in crastinum dum sanctus predicaret ostendit dicens: 'Ecce quales carnes comedit iste Franciscus quem ut sanctum honoratis! Nam mihi hoc in sero tribuit'. Sed cum membrum caponis piscis ab omnibus uideretur uelut insanus ab omni populo increpatur. Quod cum ille didicisset erubuit et ueniam postulauit; redieruntque carnes ad sui speciem postquam rediit preuaricator ad mentem» (Stella 2007, 1152-1155).

88 Montanari 2015, 110-112 riassume l'evoluzione delle usanze alimentari riguardanti il pesce: da un'iniziale esclusione dalla dieta quaresimale, il pesce viene dapprima ammesso (cioè non esplicitamente vietato) e quindi esplicitamente assimilato a ogni altro alimento 'magro', tanto è vero che dal IX-X secolo non vi era alcun dubbio sulla sua ammissibilità in Quaresima. Questo nonostante che le ovvie difficoltà di trasporto e conservazione nel Medioevo lo rendessero un cibo ricercato e costoso. Abelardo segnalò l'ipocrisia e consigliò ad Eloisa (nella VIII lettera dell'epistolario) di preferire la carne, più nutriente, meno costosa e più facile da preparare (cfr. McLaughlin 1956, 242-297): «Ea itaque sumenda sunt, quae naturae necessitas quaerit, et non quae edendi libido suggerit. Minori vero desiderio concupiscimus quae minus pretiosa esse videmus, et quae magis abundant, et vilius emuntur; sicut est communium 
L'uso alimentare dei quadrupedi, invece, non verrà considerato nella letteratura agiografica se non eccezionalmente, come nei racconti che seguono a illustrare la differenza delle punizioni conseguenti il furto di cibo tra Alto e Basso Medioevo, quando l'uccisione di una pecora aveva una drammaticità attenuata, in un contesto economicamente molto più florido. Gregorio di Tours narra di un diacono che aveva rubato le pecore della chiesa di San Giuliano d'Alvernia dicendo «Giuliano non mangia arieti». Ed ecco che dopo poco tempo fu attaccato da una febbre fortissima: si fece buttare addosso acqua per rinfrescarsi, ma subito dal suo corpo uscì tanto di quel fumo e fetore che tutti quelli che gli erano accanto scapparono e lui stesso morì in breve tempo $^{89}$. Nel XIII secolo invece si racconta di un tale che aveva rubato

cibus carnium, qui et infirmam naturam multo validius quam pisces confortat, et minores expensas, et faciliorem habet apparatum»; «Dobbiamo dunque mangiare ciò che è richiesto dalle necessità della natura e non ciò che suggerisce la libidine della gola. Di fatto però noi desideriamo con minor intensità i cibi che sappiano meno prelibati e quelli più comuni e meno costosi, come la normale carne che è più nutriente dei pesci per chi è debole e che, oltre a costare meno, è più facile a prepararsi» (trad.it. Roncoroni 1974, 348). Cfr. Zug Tucci 1985, $293-322$ e Montanari 1993, 61, 31-38. Naturalmente era anche sottesa tutta la simbologia del primo Cristianesimo, a partire

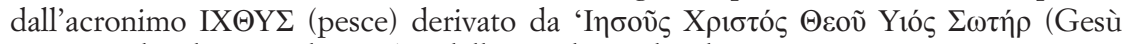
Cristo Figlio di Dio Salvatore) e dalle parole rivolte da Gesù a Pietro in Lc. 5, $10 \mu$ ஸ́

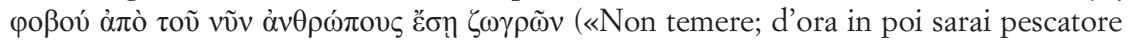
di uomini»). Questi piani di lettura sono presenti in un exemplum attestato nel f. 190r del ms. Paris, BnF lat. 16481, una raccolta di sermoni pronunciati dai frati mendicanti alla Sorbona, dove si legge di un chierico lussurioso, ma assai devoto alla Vergine, alla quale si rivolgeva regolarmente con preghiere e genuflessioni, il quale un giorno, tentato dal demonio, uscì per accompagnarsi con una ribalda, fermandosi però a pregare in una cappella e addormentandovisi. Si tratta di un tema ben noto e diversamente trattato nella letteratura agiografica: qui la Vergine compare in sogno al chierico mostrandogli un pesce squisito, ma servito in un piatto orribile e pieno di fango: la preghiera è come un cibo squisito, ma bisogna pur pulire il recipiente in cui viene servito. In questo caso il pesce è sia simbolo di un alimento ricercato e raffinato, sia immagine di purezza.

89 Gregorius Turonensis, De gloria martirum, II 17 (MGH Script. Merov. I, 2, 121-122), qui citato dalla Legenda aurea, cit., XXX 21-24, ed. cit., 246-7: «Inter cetera huius sancti miracula solet referri quod cum quidam diaconus oues ecclesie sancti Iuliani raperet et pastores eidem ex parte sancti Iuliani prohiberent, ille respondit: 'Iulianus non comedit arietes'. Et ecce, post modicum febre uehementissima perurgetur et inualescente febre quod a martyre incenditur confitetur; fecitque super se iactari aquam ut refrigeraretur, sed statim tantus fumus et fetor de corpore eius exiit ut cuncti qui aderant fugerent et ipse post modicum expirauit». 
una pecora del suo vicino e se l'era mangiata. San Patrizio aveva più volte esortato il ladro, chiunque egli fosse, a risarcire il danno, ma nessuno si faceva avanti. Mentre tutto il popolo era radunato in chiesa, ordinò nel nome di Cristo che colui nel cui ventre era entrata la pecora si mettesse a belare di fronte a tutti. Il che avvenne puntualmente: il reo fece allora penitenza, mentre tutti gli altri si astennero dai furti ${ }^{90}$. Certo rubare a una chiesa è differente che rubare a un privato cittadino, ma è evidente una certa sproporzione tra il morire fumiganti e puzzolenti e il sostanziale pater ave gloria con cui il goloso ladro irlandese se la cava.

$\mathrm{Si}$ è narrato prima di sant'Epifanio e del vino convertito in acqua. Alla fine del XII secolo Cesario di Heisterbach narra invece di sant'Arnoldo che, al servizio di un nobile che si era ripromesso di digiunare a pane e acqua tutti i sabati e tutte le festività della Vergine, trasformava inconsapevolmente in vino, semplice strumento della volontà della Madre di Dio, l'acqua nella ciotola che porgeva al penitente, con grande sconcerto e irritazione di quest'ultimo ${ }^{91}$.

90 Jacobus de Voragine, Legenda aurea, XLIX 5-9: «Quidam uir conuicini sui ouem furatus erat et comederat et cum sanctus sepius hortaretur satisfacere furem quicumque ille esset et nullus compareret, dum totus populus ad ecclesiam conuenisset, in uirtute Ihesu Christi precepit ut in cuius uentrem ouis intrasset coram omnibus balatum daret. Quod factum est et egit reus penitentiam et alii a furtis sibi cauebant. Erat etiam ei consuetudo omnes cruces quas uidebat deuote uenerari, sed ante quandam pulchram crucem inuisam preteriit et, a suis admonitus a deo cur non uiderit precibus dum requireret, audiuit uocem de sub terra: 'Non uidisti quia ego hic sepultus sum paganus et signo crucis indignus?'. Vnde fecit crucem abinde tolli» (Stella 2007, 368369). Iacopo prende questo exemplum dal Liber epilogorum in gesta sanctorum del suo confratello Bartolomeo da Trento (LXXVII 11-6, ed. Paoli 2001, 84).

91 Caesarius Heisterbacensis, Dialogus miraculorum VII 38: «Solitus erat [scil. Walterus de Birbech] in vigiliis omnium sollemnitatum beatae Virginis ieiunare in pane et aqua, et saepe etiam in sextis feriis, ob reverentiam sabbati. Die quadam servus [scil. sanctus Arnoldus] ieiunanti vas parvulum fictile cum aqua obtulit, quam in eius manibus divina virtus in vinum optimum convertit. Qui cum gustasset aquam vinum factam, servum secrete vocavit et arguit, dicens sibi vinum pro aqua ministratum. Quod cum ille negasset, asserens se hausisset aquam de situla, vas recepit, et effudit, sicque aqua purissima impleta, ut securus esset, praegustavit, et obtulit. De quo cum gustasset, sicut prius vinum invenit, et mox servo iratus sic ait: 'Ut quid mihi illudis en secundo, vinum pro aqua mihi offerendo?'. Ad quod verbum stupens servus, cum iuramentis maximis negavit. Tunc primum intelligens ex multa misericordia Dei Genitricis hoc miraculum circa se gestum, eundem servum adiuravit, ne illud unquam alicui hominum quoad viveret, manifestaret»; «Era solito [scil. Walter da Birbech] alla vigilia di tutte le feste della Beata 
Per quanto riguarda il vino nella tradizione occidentale, vanno anche considerati i racconti che mostrano come fosse un mezzo per trasmettere e anche trasportare le virtù miracolose delle reliquie del santo, qui già citati per quanto riguarda san Francesco. In Francia e in Inghilterra nei secoli XI e XII era diffusa la pratica del vinage, la preparazione di una bevanda miracolosa prodotta immergendo nel vino le reliquie di un santo o anche utilizzando l'acqua con cui se ne era lavata la tomba ${ }^{92}$. Nei Miracles de saint Benoît della seconda metà del XII secolo si parla del villaggio di Sancerre nella cui piazza durante un'epidemia vennero poste le reliquie di san Benedetto e di san Mauro: vi fu versato sopra del vino che gli abitanti raccoglievano e bevevano più che potevano, per evitare la malattia o riacquistare la salute ${ }^{93}$. Ma del vino come mezzo per ingerire la povere delle reliquie si parla anche nella zona di Laon per santa Anstrude ${ }^{94} \mathrm{e}$ in molte altre zone della Francia.

Non solo, nei racconti agiografici domenicani, l'eccessiva astinenza dal cibo è un peccato, che stronca la forza richiesta ai frati nelle loro

Vergine digiunare a pane e acqua, e spesso anche il sabato, per reverenza verso quel giorno. Una volta il suo servo [scil. sant'Arnoldo] portò a lui che digiunava una piccola tazza d'argilla con dell'acqua che la divina potenza convertì in ottimo vino. E quello assaggiando l'acqua trasformata in vino, chiamò a parte il suo servo e lo accusò di avergli dato da bere del vino anziché dell'acqua. Il servo negò dicendo di aver preso l'acqua dal secchio, si riprese la tazza, la svuotò e dopo averla riempita di acqua purissima la assaggiò per sicurezza e la porse. E quando quello la assaggiò, trovò che era vino come prima e irato gli disse: 'Mi prendi ancora in giro una seconda volta, dandomi da bere vino per acqua?'. Il servo stupito a quelle parole negò assolutamente con i giuramenti più sacri. Allora capendo improvvisamente che questo era un miracolo fatto per lui dalla grande misericordia della Madre di Dio, scongiurò lo stesso servo di non rivelare mai ad alcun uomo quello che era successo, finché fosse vissuto» (Strange 1851, II 52).

92 Verdon 2002, 274-277.

93 Citato in Verdon 2002, 275.

94 Vita Anstrudis abbatissae 29, ed. MGH Script. rer Merov. VI 76: «Quaedam vero ex sororibus fluxum sanguinis patiebatur gravissimum. Quae vidit in somnis quandam monacham precipientem sibi, ut medicinam aliam nequaquam sumeret, nisi rasum pulverem de sepulchro sanctae virginis [scil. sanctae Anstrudis] in vino biberet calido. Quae evigilans, iussa complevit atque in ipso momento a fluxu sanguinis sana effecta est»; «Una delle consorelle soffriva continuamente di una gravissima emorragia. E vide in sogno una monaca che le ordinò di non prendere altra medicina se non bere nel vino caldo della polvere raschiata dal sepolcro della santa vergine [scil. santa Astrude]. E svegliatasi fece quello che le era stato prescritto e in quello stesso momento fu guarita dal flusso di sangue». Citato da Montanari 2015, 215, n. 42. 
attività. Il domenicano Stefano di Borbone nel XIII secolo racconta che i suoi confratelli delle origini si maceravano troppo con digiuni e privazioni e che il diavolo ne approfittava per ossessionarli. È infatti lo stesso diavolo ad invitare a una astinenza smodata, cosicché i frati divengano inutili e di peso per se stessi e per gli altri, togliendo a Dio e ai confratelli l'aiuto che potrebbero e dovrebbero dare ${ }^{95}$. Nelle stesse pagine ancora lo stesso frate predicatore racconta del diavolo che rimase malissimo quando Maria d'Oigny interruppe, mettendosi a mangiare, il digiuno che la stava fiaccando ${ }^{96}$. Allo stesso modo Filippo da Ferrara, frate predicatore

95 Stephanus de Borbone, Tractatus de diversis materiis praedicabilibus, III 5: «Et nota quod indiscreta abstinentia facit aliquando homines in religione impotentes et inutiles, quod sint ibi graues et onerosi et aliis et quod non possint exequi officia sua ad que sunt uocati [...] Item per hanc rapit et aufert Deo uel fratribus suis adiutorium quod eis facere debebat. [...] QUOMODO DYABOLUS MONET INDISCRETAM ABSTINENTIAM. Videns autem dyabolus in principio ordinis Predicatorum fratres nimis affligi paupertate et defectu cibi et sompni, et cum multum affligeret eos, etiam corporaliter, fratres statuerunt ut in fine matutinarum cantarent 'Te Sanctum Dominum', ut boni angeli quos inuocabant malorum nocumentum reprimerent. Clamauit dyabolus per os cuiusdam fratris, quem propter indiscretam abstinentiam arripuerat, cum cantarent 'Chrubin quoque ac Seraphin', etc.: 'O miseri! uos nescitis quid cantatis. Nescitis quam sublimes isti sunt, sed ego scio, qui de eorum consortio decidi; et cum non habeam carnem in qua possim facere penitentiam, non possum illuc ultra conscendere; sed certe, si tantum haberem de carne quantum est in pollice humano, tantum facerem in ea de penitentia, quod adhuc ad altiorem statum ascenderem'. Magister Iordanus, uidens demonis fallaciam, mouebat fratres contra indiscretam abstinentiam»; «E si noti che l'astinenza smodata rende talvolta gli uomini che hanno preso i voti impotenti e inutili, cosicché sono di peso a se stessi e agli altri e non possono eseguire i compiti a cui sono chiamati. [...] E così per mezzo di questa astinenza colui che se ne affligge ruba e sottrae a Dio o ai suoi confratelli l'aiuto che deve prestare loro [....]. IN CHE MODO IL DIAVOLO INCITA ALLA ASTINENZA SMODATA Vedendo dunque il diavolo che agli inizi dell'Ordine dei Predicatori i frati erano molto afflitti dalla povertà e dalla mancanza di cibo e di sonno e molto affligendoli, anche fisicamente, i frati stabilirono di cantare alla fine del mattutino 'Te Sanctum Dominum', cosicché i buoni angeli che invocavano scacciassero le aggressioni di quelli malvagi. Il diavolo allora si mise a gridare per bocca di un frate, che aveva ossesso a causa della di lui smodata astinenza, mentre cantavano 'I Cherubini e i Serafini ecc.': 'O Miseri! Voi non sapete che cosa cantate. Non sapete quanto siano sublimi costoro, ma lo so io, che sono decaduto dal loro consozio; e dato che non ho carne su cui possa fare penitenza, non posso più risalire; ma sicuramente se avessi anche solo la carne che c'è in un pollice umano, farei su di essa tanta di quella penitenza da salire anche più in alto'. Il maestro Giordano, vedendo la fallacia del diavolo, ammonì allora i frati contro l'astinenza smodata» (Berlioz 2006, 190-191). 96 Stephanus de Borbone, Tractatus de diversis materiis praedicabilibus, III 5: 
del primo XIV secolo, racconta che l'eccessiva astinenza dal cibo è un peccato: il maestro Giordano di Sassonia, tentato dal diavolo, che gli si era presentato sotto forma di un uomo timorato di Dio, si astenne completamente dalla carne, e divenne così debole da non riuscire più a stare in piedi. Dio gli rivelò infine che si trattava di suggestione diabolica ${ }^{97}$.

«Item, in Vita Marie d'Ognies dicitur quod cum multo ieiunio se afflixisset et comederet ne corpus deficeret, apparuit dyabolus dicens: 'Comede, gulosa!', plus dolens de comestione quam abstinentia eius»; «Allo stesso modo, nella Vita di Maria d'Ognies si dice che dopo essersi macerata con un grande digiuno stava mangiando perché il suo corpo non venisse meno e il diavolo gli apparve dicendole: 'Mangia, ingorda!', più addolorato dal fatto che stesse mangiando di quanto lo fosse stato per il digiuno» (Berlioz 2006, 191).

97 Gerardus de Fracheto, Vitae Fratrum, III 28: «Temptavit eum malignus semel, cum esset Parisius infirmus, sub mira fraudulancia sanctitatis; veniens enim ad portam in forma venerande persone, peciit se ad magistrum Iordanem deduci. Cum ergo ductus cum eo aliquos habuisset familiares sermones, postulavit fratres secedere, quasi secreto locuturus cum eo. Quo facto sic ait: 'Magister tu es caput huius sanctissimi ordinis universorum fratrum; quod si parvum vel grande quid a te exierit contra religionis fervorem relaxacionis vestigium, cum natura humana prona sit ad declinandum, tu exinde penam reportabis a domino, et in tanto ordine relinques exemplum dissolucionis et turbacionis materiam; es enim infirmus, sed non intantum, ut non possis carere culcitra et a carnibus abstinere. Quod si cras vel post cras non fiat eadem dispensacio fratri plus vel eque infirmo, fiet inde iudicium, murmur et turbacio orietur. Unde rogo et consulo, quod sicut actenus fuisti religionis exemplum in aliis, ita te prebeas et in istis'. Sic ergo versipellis ille colorans licenciatus recessit, ruminando aliquid ac si diceret psalmos. Credidit homo Dei simpliciter verbis eius, et abstinuit multis diebus ab illis. Unde intantum crevit eius debilitas, quod vix sustinere valebat. Revelatum est autem ei a domino quod, qui illa suggesserat, dyabolus erat, qui eius vite et eius predicacionibus invidebat»; «Una volta venne [scil. Giordano di Sassonia] tentato dal Maligno, mentre era ammalato a Parigi, sotto un sorprendente infigimento di santità. Infatti si presentò alla porta sotto le spoglie di una persona veneranda e chiese di essere condotto dal maestro Giordano. E condotto da lui, dopo aver scambiato qualche parola familiare, chiese ai confratelli di allontanarsi, come se volesse parlare segretamente con lui. E quando si fece così disse: 'Maestro, tu sei il capo di tutti i frati di questo grandissimo ordine; per cui, qualsiasi cosa, piccola o grande, venga da te che possa essere un segno di rilassatezza contro il fervore religioso, essendo la natura umana incline al cedimento, tu ne risponderai davanti al Signore, lasciando in un Ordine tanto grande un esempio di dissoluzione e materia di turbamento. Tu sei infatti ammalato, ma non così tanto da non poter fare a meno del materasso e da non poterti astenere dalle carni. E se domani o dopodomani non ci sarà la stessa dispensa per ogni confratello ammalato più gravemente o nello stesso modo, ne verranno critiche e mormorii e ne nascerà turbamento. Quindi ti chiedo e ti consiglio che come finora sei stato esempio di rigore religioso in altre cose, così ti mostri anche in queste'. Con queste parole ingannatrici allora quel 
A quanto sembra nel tardo Medioevo è un vero peccato, in un altro senso, astenersi dal cibo anche quando questo è preparato dal demonio in persona. Jacques de Vitry, all'inizio del XIII secolo racconta di aver visto un monaco della Chartreuse assalito da una fame terribile prima dell'ora del pasto nella sua cella. Mentre era quasi incapace di pregare per questo attacco di fame, vide Satana sotto l'aspetto di una graziosa fanciulla che si dava da fare vicino al focolare cucinando e mettendo legna sul fuoco. Alla fine la ragazza gli servì un succulento piatto di legumi e scomparve. Rimasto solo davanti alla scodella, il monaco moltiplicò le preghiere e riuscì ad arrivare all'ora canonica del pranzo senza toccarla. Chiese allora al priore che cosa doveva fare di quel piatto preparato dal demonio. Il priore rispose che non c'è nulla che Dio abbia creato che debba essere rifiutato in stato di grazia. Dunque il monaco mangiò e dichiarò che mai, da laico o da religioso, aveva mai mangiato un cibo così ben preparato e così buono ${ }^{98}$.

Un diverso approccio culturale verso il cibo e alle dinamiche connesse all'alimentazione nella letteratura agiografica nell'Alto Medioevo e nei secoli seguenti è riconoscibile anche nella figura agiografica legata alla diffusione della birra, che inizia a essere prodotta a partire dal IX$\mathrm{X}$ secolo, soppiantando la cervogia di solo orzo senza luppolo. La cervogia, una sorta di porridge più liquido, era mal considerata: ancora nella vita di san Luigi si racconta come il santo ne facesse un uso penitenziale in Quaresima, per sostentarsi. Il patrono dei birrai, sant'Arnolfo di Soissons, ebbe invece una parte attiva nell'XI secolo a incoraggiare la diffusione della birra, avendo costatato la maggiore resistenza durante un'epidemia dei bevitori di birra rispetto ai bevitori d'acqua". A questo proposito, oltre alla gradazione alcolica, va sottolineato il fatto che la produzione della birra richiede l'ebollizione. La sua leggenda narra appunto come sant'Arnolfo avesse utilizzato il suo bastone d'abate durante la preparazione della birra ${ }^{100}$. È dunque a una

traditore camuffato congedato se ne andò, biascicando qualcosa come se stesse recitando i salmi. L'uomo di Dio credette nella sua semplicità alle sue parole e si astenne per molti giorni da quelle cose. E la sua debolezza aumentò così tanto che poteva a malapena alzarsi. Allora i Signore gli rivelò che chi gli aveva suggerito quelle cose era il diavolo, che provava invidia per la sua vita e la sua attività di predicatore» (Reichert 1897, 122-123).

98 Jacobus Vitriacensis, Historia Occidentalis, 18, cfr. Hinnebusch 1972, 123-124.

99 Moulin 1984, 13-31.

100 Ferraut 1887. 
figura agiografica che viene attribuita una variazione decisiva per il gusto e l'apprezzamento di una bevanda.

Si è detto che il racconto agiografico, soprattutto a partire dal XII secolo, non è in sé autonomo, ma è parte di un sistema di comunicazione che ha come elemento complementare la predicazione, in genere orientata da sermoni modello che lo stesso agiografo predisponeva. Il destinatario finale delle leggende agiografiche inserite in questo sistema di comunicazione era in genere il pubblico laico, per cui ogni leggenda era scomposta per presentare elementi dottrinali ed etici, riferendosi alla vita quotidiana e a riferimenti culturali che si sapevano condivisi. Non sorpenderà quindi il fatto che nei Sermones de sanctis di Iacopo da Voragine, il cibo non abbia mai una funzione negativa e che compaiano, sia pure con riferimenti biblici, immagini culinarie come il Cristo, presentato come il lardo in una padella (a commentare il salmo Ossa mea ut cremium arens ${ }^{101}$. O l'anima come una cucina, da dove vanno scacciati i cani ${ }^{102}$. È lo stesso contesto culturale quasi opposto al contemptus mundi monacale e alla mortificazione del corpo che fa sì che l'agiografo ritenga significativo raccontare che san Francesco prima di morire chiese di mangiare i mostaccioli (dolci di mandorle e zucchero) preparati da una nobildonna

101 Cfr. Jacobus de Voragine, Sermones Quadragesimales, f. IV II hebd., s. II: «Et item: 'Ossa mea sicut cremium aruerunt'. Cremium est lardum in sartagine adustum et aridum, de quo omnis pinguedo exivit. Corpus ergo Christi fuit cremium, quia in sartagine crucis sic fuit igne passionis adustum quod totus sanguis de eo exivit, et in cruce sicut lignum aridum remansit»; «E ancora Ps. 102.4 'Ardono come braci le mie ossa'. La brace è il lardo bruciato e disseccato in padella, da cui è uscito tutto il grasso. Il corpo di Cristo fu dunque brace, poiché nella padella della croce fu così bruciato dal fuoco della passione che ne uscì tutto il sangue e rimase in corce come legno arido» (Maggioni 2005, 154). Cfr. anche Jacobus de Voragine, Sermones de sanctis. Volumen breve, De purificatione virginis: «Christus in nostrum obsequium consumpsit $[\ldots]$ sanguinem per effusionem, Ps.: 'Ossa mea sicut cremium aruerunt'. Sicut enim in cremio nihil remanet pinguedinis. sic et in corpore Christi nihil remansit sanguinis»; «Cristo in nostro onore sacrificò il proprio sangue per effusione, Ps. 102, 4: 'Ardono come braci le mie ossa'. Come infatti nella brace non rimane nulla del grasso della carne, così anche nel corpo di Cristo non rimase nulla del suo sangue» (Maggioni c.d.s.).

102 Jacobus de Voragine, Sermones Quadragesimales, Dom. I s. II 35: «Sicut aqua bulliens fugat canem de coquina, sic oratio fervens fugat diabolum de anima»; «Come l'acqua bollente scaccia il cane dalla cucina, così un'orazione fervente scaccia il diavolo dall'anima» (Maggioni 2005, 55). 
romana ${ }^{103}$ e che fra' Ginepro abbia una volta costretto il ministro generale dell'Ordine ad assaggiare nottetempo una farinata al burro apostrofandolo: «Se non la mangi tu, tienmi la candela che la mangio io!» $\gg^{104}$. Rispetto all'agiografia altomedievale, nei testi del basso Medioevo emerge dunque il riflesso di un concetto mutato di corpo, di un' idea diversa della socialità nella somministrazione e nella consumazione del cibo.

\section{BIBLIOGRAFIA}

BARTELINK 1974 - Palladio, La storia Lausiaca, a cura di G.J.M. Bartelink, Milano, Mondadori (Fondazione Lorenzo Valla), 1974.

BARTOLI 1980 - M. Bartoli, Analisi storica e interpretazione psicanalitica di una visione di S. Chiara d'Assisi, Archivum Franciscanum Historicum 73 (1980), 449-472.

BASTANZI 1988 - G. Bastanzi, Le superstizioni delle Alpi venete, Treviso, Tipografia Luigi Zoppelli, 1988.

BAUTIER 1984 - A.M. Bautier, Pain et patisserie dans les textes mediévaux latins antérieurs au XIIIe siècles, in D. Menjot (éd.), Manger et boire au Moyen Age, Actes du Colloque de Nice (15-17 octobre 1982), Paris 1984, 33-65.

BERLIOZ 2006 - Stephanus de Borbone, Tractatus de diversis materiis predicabilibus, III, De eis que pertinent ad donum scientie et penitentiam, éd. J. Berlioz, Turnhout, Bepols, 2006.

Blangez 1979-1986 - G. Blangez (éd.), Ci nous dit. Recuil d'exemples moraux, Paris, SATF, 1979-1986.

Bynum 1982 - C.W. Bynum, Jesus as mother: studies in the spirituality of the High Middle Ages, Berkley-Los Angeles- London, University of California Press, 1982.

CASAgRAnde-VeCchio 2000 - C. Casagrande - S. Vecchio, I sette vizi capitali. Storia dei peccati nel Medioevo, Torino, Einaudi, 2000.

Casio-Messa 2015 - G. Casio - P. Messa, Il cibo di Francesco. Anche di pane vive l'uomo, Milano, Edizioni Terra Santa, 2015.

Cecchini 2004 - Uguccione da Pisa, Derivationes, a cura di E. Cecchini et alii, Firenze, SISMEL Edizioni del Galluzzo, 2004.

CHIESA 1983 - P. Chiesa, Ambiente e tradizioni nella prima redazione latina della leggenda di Barlaam e Josaphat, Studi medievali 24 (1983), 521-544.

ChIESA-Dolbeau 1989 - P. Chiesa - F. Dolbea, Una traduzione amalfitana dell'XI secolo: la Vita latina di sant'Epifanio, SM 30 (1989), 909-951.

CignitTi 1967 - B. Cignitti, s.v. Mama di Cesarea, in Bibliotheca Sanctorum, Roma, Città nuova, 1961, VIII, coll. 591-611.

103 Compilatio Assisiensis, 8, in Menestò-Brufani-Cremascoli 1995, 1477. Cfr. Cassio-Messa 2015, 55-57.

104 Petrocchi 1960, citato in Cassio-Messa 2015, 76-77. 
Citati-Lilla 1974 - Atanasio, Vita di Antonio, a cura di G.J.M Bartelink - P. Citati - S. Lilla (traduzione), Milano, Mondadori (Fondazione Lorenzo Valla), 1974.

COLES 1995 - A. Coles, Biomedical models of reproduction in the fifth century BC and Aristotle's Generation of animals, Phronesis 40 (1995), 48-88.

Cross 1969 - P. Cross, Motif-Index of early irish literature, Bloomington, Indiana University, 1969.

Dalarun 2009 - J. Dalarun, "Sicut mater". Una rilettura del biglietto di Francesco d'Assisi a frate Leone, Frate Francesco 75 (2009), 19-51 (=J. Dalarun, «Sicut mater». Une relecture du billet de Françoise d'Assise à frère Leon, Le Moyen Age 113, 2007, 639-668).

DAPELO 2001 - G. Dapelo, Il romanzo latino di Barlaam e Josaphat (BHL 979): preparando l'edizione, Filologia Mediolatina 8 (2001), 179-220.

Delaisi de Parseval 1981 - G. Delaisi de Parseval, La part du père, Paris, Seuil, 1981. Dindorf 1859 - Epiphanii Episcopi Constantiae Opera, ed. W. Dindorf, Leipzig, 1859.

Balthazar Bellerus 1624 - Vincentius Bellovacensis, Speculum quadruplex sive Speculum maius, I, Speculum Historiale, ed. Balthazar Bellerus, Douai, 1624.

Dumville 1976 - D.N. Dumville, Echtrae and Immram: some problems of definition, Ériu 27 (1976), 82-83.

ECO 2009 - U. Eco, Introduzione al Medioevo, in U. Eco (a cura di), Il Medioevo, Milano, Bompiani, 2009, I, 14-87.

FERRAUt 1987 - S. Ferraut, Vie de Saint Arnold ou Arnulphe, éveque de Soissons, Bruges, Bayaert-Storie, 1887.

Fiorentino 2001 - Tommaso d'Aquino, Il male, a cura di F. Fiorentino, Milano, Bompiani, 2001.

GRICOURT 1957a - J. Gricourt, A propos de l'allaitement symbolique: le domaine irlandais, in Hommages à Waldemar Deonna (Collection Latomus XXVII), Bruxelles, Latomus, 1957, 249-257.

GRICOURT 1957b - J. Gricourt, L'oreille droite de saint Fraech, Ogam 9 (1957), 187-194.

Hinnebusch 1972, The historia occidentalis of Jacques de Vitry. A critical edition, ed. J.F. Hinnebusch, Fribourg, Fribourg University Press, 1972.

JABOUlET-VERCHERRE 2014 - A. Jaboulet-Vercherre, The fbysician, the drinker, and the drunk: wine's uses and abuses in Late Medievak natural philosophy, Turnhout, Brepols, 2014.

LiONETTI 1988 - R. Lionetti, Le lait du père, Paris, Imago, 1988 (Latte di padre, Brescia, 1984).

Mac NéILl 1921 - E. Mac Néill, Celtic Ireland, Dublin, Parsons, 1921.

MAGgIONI 2005 - Iacopo da Varazze, Sermones Quadragesimales, a cura di G.P. Maggioni, Firenze, SISMEL Edizioni del Galluzzo, 2005.

MagGIONI 2013 - Jean de Mailly, Abbreviatio in gestis sanctorum, a cura di G.P. Maggioni, Firenze, SISMEL Edizioni del Galluzzo, 2013.

Maggioni-Taviani-Tinti c.d.s. - G.P. Maggioni - P. Taviani - R. Tinti, Il Purgatorio di san Patrizio, Firenze, c.d.s. (Quaderni di Hagiographica)

Marcovich 2002 - Clemens Alexandrinus, Paedagogus, ed. M. Marcovich, LeidenBoston, Brill, 2002.

MCLAUGHLIN 1956 - M.M. McLaughlin, Abelard's rule for religious women, Mediaeval Studies 18 (1956), 241-292. 
Menestò-Brufani-Cremascoli 1995 - Fontes Franciscani, a cura di E. Menestò - S. Brufani, Assisi, Edizioni Porziuncola, 1995.

Montanari 1993 - M. Montanari, La fame e l'abbondanza. Storia dell'alimentazione in Europa, Roma-Bari, Laterza, 1993.

Montanari 2015 - M. Montanari, Mangiare da cristiani, Milano, Rizzoli, 2015.

Moulin 1984 - L. Moulin, La Bière, une inventuon médévale, in D. Menjot (éd.), Manger et boire au Moyen Age, Actes du Colloque de Nice (15-17 octobre 1982), Paris, Les Belles Lettres, 1984.

Nardo Cibele 1887 - A. Nardo Cibele, Acque. Pregiudizi e leggende bellunesi, Archivio per lo studio delle tradizioni popolari 6 (1887), 575-592.

PAOLI 2001 - Bartolomeo da Trento, Liber epilogorum in gesta sanctorum, a cura di E. Paoli, Firenze, Edizioni del Galluzzo, 2001.

Petrocchi 1960 - La vita di frate Ginepro. Testo latino e volgarizzamento, a cura di G. Petrocchi, Bologna (Commissione per i Testi di Lingua), 1960.

Plummer 1922 - C. Plummer, Bethada Naem n-Erenn. Lives of Irish Saints, Oxford, Clarendon Press, 1922, I, 168-182. Traduzione inglese ibidem, II, 162-176.

PRICOCO 1995 - Benedetto da Norcia, La Regola di San Benedetto e le regole dei Padri, a cura di S. Pricoco, Milano, Mondadori (Fondazione Lorenzo Valla), 1995.

Pricoco-Simonetti 2006 - Gregorio Magno, Storie di santi e di diavoli, II, a cura di S. Pricoco e M. Simonetti, Milano, Mondadori (Fondazione Lorenzo Valla), 2006.

RAPP 1991 - C. Rapp, The Vita of Epiphanius of Salamis. An bistorical and literary study, Oxford, 1991.

REICHERT 1897 - Gerardus de Fracheto, Vitae Fratrum Ordinis Praedicatorum, ed. B.M. Reichert, in Monumenta Ordinis Fratrum Praedicatorum Historica, I, RomaeStuttgardiae, 1897.

RonCHEY 2012 - S. Ronchey, Il Buddha bizantino, in S. Ronchey-P. Cesaretti (a cura di), Storia di Barlaam e Ioasaf. La vita bizantina del Buddha, Torino, Einaudi, 2012, vii-cvii.

RONCORONI 1974 - Abelardo, Storia delle mie disgrazie. Lettere d'amore di Abelardo ed Eloisa, a cura di F. Roncoroni, Milano, Garzanti, 1974.

SENZAKI-REPS 1973 - N. Senzaki - P. Reps (a cura di), 101 storie zen, Milano, Adelphi, 1973.

Stella 2007 - Iacopo da Varazze, Legenda Aurea. Con le miniature del codice Ambrosiano C 240 inf., G.P. Maggioni (testo critico riveduto e commento) - F. Stella (edicizione coordinata da), Firenze, SISMEL Edizioni del Galluzzo, 2007.

STOKES 1890 - Lives of Saints from the Book of Lismore, ed. W. Stokes, Oxford, Clarendon Press, 1890

STRANGE 1851 - Caesarius Heisterbacensis, Dialogus miraculorum, ed. J. Strange, KölnBonn-Bruxelles, 1851.

Tolstoj - L.N. Tolstoj, La confessione, Milano, Feltrinelli, 2010 (trad. G. Pacini).

VAUCHEZ 2000 - A. Vauchez, Santi profeti e visionari. Il soprannaturale nel Medioevo, Bologna, Il Mulino, 2000.

VeCCHIO 2008 - Filippo da Ferrara, Liber de introductione loquendi, ed. S. Vecchio, I castelli di Yale 3 (1998), 139-165.

VECCHIO 2009 - S. Vecchio, Gusto piacere, peccato nella cultura medievale, I Castelli di Yale 10 (2009), 27-40. 
Verdon 2002 - J. Verdon, Bere nel Medioevo. Bisogno, piacere o cura, Bari, Dedalo, 2002 (Boire au Moyen Age, Paris, 2002).

WaLz 1935 - Humbertus de Romanis, Legenda sancti Dominici, éd. A. Walz, in MOPH 16 (1935), 353-433.

Zug Tucci 1985 - H. Zug Tucci, Il mondo medievale dei pesci tra realtà e immaginazione, Spoleto, CISAM, 1985, 293-322. 